\section{Pacific Northwest}

National Laboratory

Operated by Battelle for the

U.S. Department of Energy

\title{
A Case Study of Selected Photographic Inspection Techniques for a Transparency Regime
}

\author{
W.K. Pitts \\ N. Dahl \\ E. Fuller \\ B.D. Geelhood \\ R.R. Hansen \\ M. Knopf \\ R.T. Kouzes \\ C. Wyatt
}

January 2003

Prepared for the U.S. Department of Energy under Contract DE-AC06-76RL01830 


\title{
DISCLAIMER
}

This report was prepared as an account of work sponsored by an agency of the United States Government. Neither the United States Government nor any agency thereof, nor Battelle Memorial Institute, nor any of their employees, makes any warranty, express or implied, or assumes any legal liability or responsibility for the accuracy, completeness, or usefulness of any information, apparatus, product, or process disclosed, or represents that its use would not infringe privately owned rights. Reference herein to any specific commercial product, process, or service by trade name, trademark, manufacturer, or otherwise does not necessarily constitute or imply its endorsement, recommendation, or favoring by the United States Government or any agency thereof, or Battelle Memorial Institute. The views and opinions of authors expressed herein do not necessarily state or reflect those of the United States Government or any agency thereof.

\author{
PACIFIC NORTHWEST NATIONAL LABORATORY \\ operated by \\ BATTELLE \\ for the \\ UNITED STATES DEPARTMENT OF ENERGY \\ under Contract DE-ACO6-76RLO183O
}

Printed in the United States of America

\author{
Available to DOE and DOE contractors from the \\ Office of Scientific and Technical Information, \\ P.O. Box 62, Oak Ridge, TN 37831-0062; \\ ph: (865) 576-8401 \\ fax: (865) 576-5728 \\ email: reports@adonis.osti.gov
}

This document was printed on recycled paper.

$(8 / 00)$ 


\title{
A Case Study of Selected Photographic Inspection Techniques for a Transparency Regime
}

\author{
W.K. Pitts \\ N. Dahl \\ E. Fuller \\ B.D. Geelhood \\ R.R. Hansen \\ M. Knopf \\ R.T. Kouzes \\ C. Wyatt
}

January 2003

Prepared for the U.S. Department of Defense Defense Threat Reduction Agency 


\section{Table of Contents}

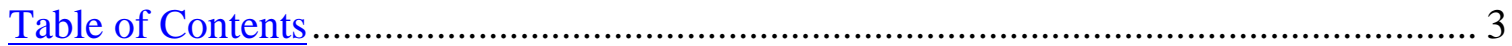

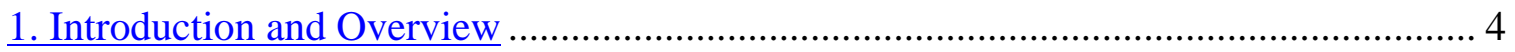

1.1 Advantages of Photographic Inspection ....................................................... 4

$1.2 \quad$ Scenarios and Precedents............................................................................. 5

1.3 Technical Enabling Tools ………….......................................................... 5

2. Example Applications and Scenarios..................................................................... 7

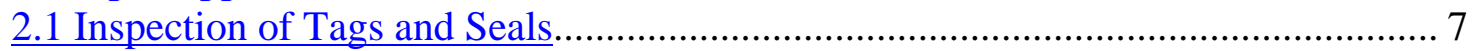

2.1.1 A Study of 3M Confirm ${ }^{\mathrm{TM}}$ Tape …………….............................................. 8

2.1.2 Inspection of Confirm ${ }^{\mathrm{TM}}$ Tape with Hand Held Cameras.................................. 9

2.1.3 Inspection of Confirm ${ }^{\mathrm{TM}}$ with a Zoom Microscope ......................................... 10

2.1.4 An Example Implementation of an Inspection System................................... 11

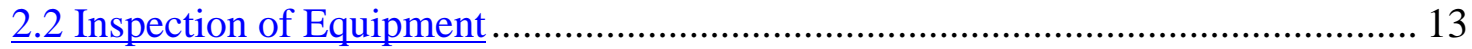

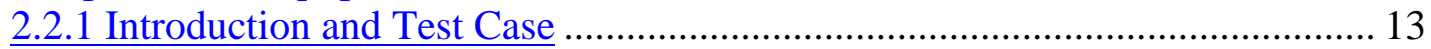

2.2.2 Example Application to Modified Circuit Boards............................................ 14

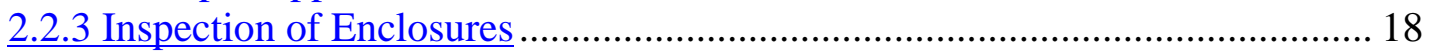

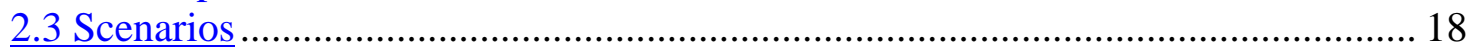

2.3.1 Photography with Monitor Control and Original Media Removal.................. 18

2.3.2 Photography With Host Approval and No Original Media Removal............... 19

2.3.3 Photography Supplied by the Host from Archival Photographs...................... 19

3. Detailed Technical Results ................................................................................. 20

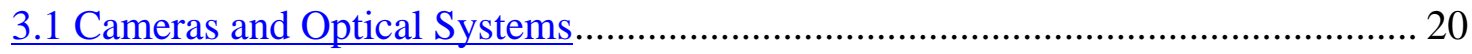

3.1.1 The Nikon Coolpix 995 Digital Camera......................................................... 20

3.1.2 The Mamiya 645 Professional Roll Film Camera ........................................... 24

3.1.3 The Polaroid MP5 Forensics Camera ........................................................ 26

3.1.4 The Thales Zoom Microscope ……………………........................................ 27

3.1.5 A Demonstration System for Floor Seals .................................................... 29

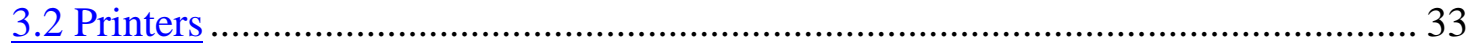

3.2.1 Generic Ink Jet Printers............................................................................ 33

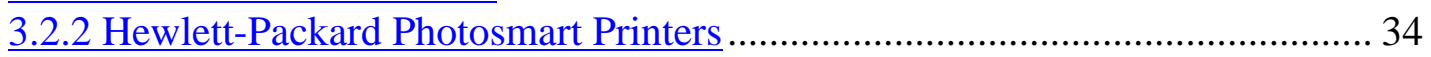

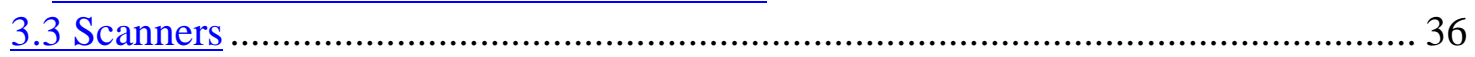

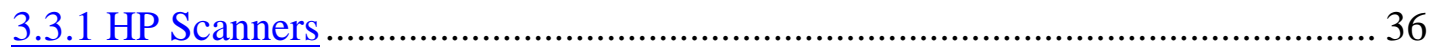

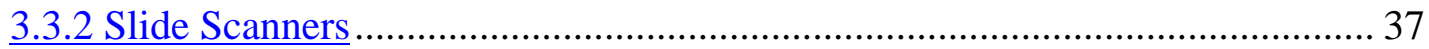

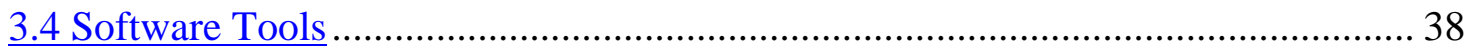

3.4.1 Photoshop ${ }^{\mathrm{TM}}$ and Color Correction ............................................................... 38

3.4.2 Change Detection System ......................................................................... 42

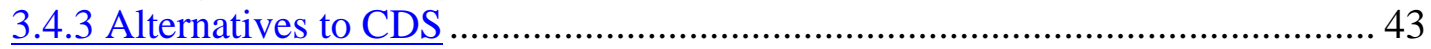

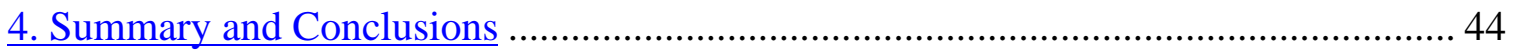

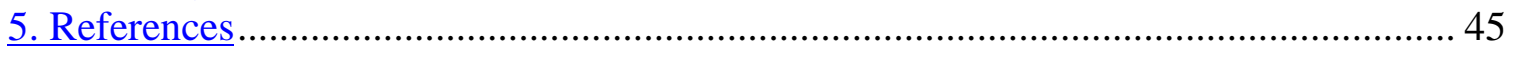




\section{Introduction and Overview}

Photographic inspection techniques have become technically more sophisticated in recent years with the development of advanced equipment for the mass consumer market. High quality digital cameras, for example, are now available around the world. Combined with an appropriate image analysis program on a personal computer, there is now the ability to produce and analyze high quality photographs with a modest level of resources.

This report is the summary of a variety of efforts, all aimed at investigating the application of commonly available, mass-market photographic and computer equipment to photographic inspection and analysis of equipment and items. It contains results of equipment test and evaluation as well as a few selected example applications.

This study was deliberately limited to examination of those techniques and equipment that would be suited to on-site inspection in a transparency regime, i.e. a possible regime in which these techniques would be disclosed and performed in a public setting of full disclosure and openness. Under these circumstances, the direction of this case study becomes an examination of simple techniques using widely available mass-market equipment.

\subsection{Advantages of Photographic Inspection}

Photographing unique items or individuals can be a simple and low-cost yet highly effective technique to distinguish an individual item or to evaluate changes of the same item with time. Wildlife biologists, for example, commonly use photographs of lions and cheetahs to identify individuals within a population. Photographic inspection of technical equipment can be a useful adjunct to other inspection techniques, but extracting the information may be problematic. The image could be too complicated, with too many individual elements, or acquiring the image may require specialized mounting jigs and lighting. New techniques have been developed to perform sophisticated photographic inspection and comparison of objects, simplifying the inspection process and enabling a higher degree of image interpretation. This case study is a report on photographic analysis applied to change and tamper detection of items within a population and possible implementation of photographic inspection techniques for a realistic scenario using tags and seals.

Photographic inspection is a technique to preserve and archive visual observation, furnishing a technical record that is easily understood by the non-specialists, using tools that are easily understood and operated by non-technical personnel. Photographs are commonly introduced as evidence of an event, allowing any observer to view the scene as an eyewitness: a photograph is typically regarded as proof of an event. Visual observation and analysis is one of the few analytical techniques that are understood by the non-specialist. 


\subsection{Scenarios and Precedents}

The techniques presented in this report are intended for an open, transparent environment where the site operator may restrict allowed techniques. Rather than develop the most technically advanced and sophisticated equipment and techniques, the strategy has been the development of simple techniques, suitable for mass-market equipment to be used by non-technical inspectors. For example, one scenario would be that an observer would direct site personnel to make a photograph of a seal, to be furnished to the observer upon exiting the site. Restricting the equipment to generally available equipment does result in lost capabilities; for example, infra red or thermal imaging would be a desirable technique. However, there are no mass-market imaging devices, and procuring the equipment may prove problematic due to cost, availability, and security concerns associated with certain equipment classes.

There is a history of photographic inspection in various inspection and arms control regimes. While it is commonly applied as a unilateral inspection method from a satellite or aircraft, photographs are commonly used in several current and past regimes:

- Photography was an option allowed under the INF Treaty, recording ambiguous situations for further analysis.

- The Cobra optical loop seal is analyzed by comparison of before and after photographs.

- The E-cup seal, commonly used by the IAEA, is verified by comparing photographs of scribed patterns, recorded at application and removal.

- Video surveillance is a common tool at many facilities, including both live and recorded video.

- Conventional weapons have been successfully characterized by photographs of intrinsic markings such as weld and casting marks on tank turrets.

\subsection{Technical Enabling Tools}

The traditional tools of photographic analysis are primarily analog (film) cameras and enlargers, supplemented by specialty equipment such as stereoscopes and blink comparators. Photographic inspection tools are now much more widely applicable due to recent advances in digital scanners, high-resolution scanners, and photographic-quality printers. An important advantage of this equipment is that high quality equipment, fully comparable to professional quality equipment several years ago, is now available from consumer mass-market sources.

Digital cameras: digital cameras are now available with sufficient resolution to make large format prints. Resolution is a function of both the image size (i.e. 2 megapixel) and the optical system of the camera. While digital cameras still do not compare with the best analog (film) cameras, digital cameras have the advantage of ease of use, immediate production of the image, large data capacity, and ability to quickly share data by copying storage media. Even though most models are relatively simple "point and shoot" snapshot cameras, several cameras are well suited for good macro (close up) photography 
of small items such as tags and seals. The results in this report were usually acquired with the Nikon Coolpix 995, a high-end mass-market camera with a good macro capability. There are many other equivalent models from other vendors.

Scanners: scanners are required to convert a print into a digital file. Scanners are now advertised with up to 2400 dpi capability. The results in this report were usually acquired using either a Hewlett-Packard 5300c or 7400c scanner. There are many other equivalent models from other vendors. In addition, there are professional scanners that can scan photographic negatives with very high resolution for professional quality prints.

Photographic quality printers: printers are required to produce an image from a file. During this study several printers were investigated, since there may be situations in which removing the image may be problematic. If there is a sensitivity toward removing either or both a magnetic storage card and a physical image from a particular room or facility, one possible image removal technique would be to print out the contents of the storage card and then scan the resulting images in a different location. Several massmarket printers were evaluated in this study, primarily Hewlett-Packard Photosmart printers with the capability to directly read a camera's storage card. There are other equivalent models from other vendors.

Image analysis software: analysis programs such as Adobe Photoshop ${ }^{\mathrm{TM}}$ have been developed to give a photographic analysis and manipulation capability that goes far beyond any darkroom technique for prints. It is simple, for example, to match the color of a photograph to a true color strip using Photoshop ${ }^{\mathrm{TM}}$ and a test strip. The best of these programs are typically research quality programs that allow scientific analysis of images. Even the simpler programs such as Microsoft Paint allow an image to be enlarged and its properties adjusted to highlight features.

Blink comparison: A further class of image analysis is blink comparison, a method to rapidly compare two aligned images and detect any features not in common between the two images. The classic application is blink comparison of astronomical photographs, in which two time-separated photographs are aligned using the fixed stars common to both images. After alignment of the two photographs, the two images are rapidly interchanged. The fixed stars are common to both images and are seen to have no apparent motion. Objects moving against the fixed stars flicker in position or intensity between the two images and are easily distinguished. Amateur astronomers have used blink comparison techniques to discover asteroids even with small backyard telescopes. [1] Blink comparison works best with an unchanging background having similar lighting; astronomical photographs of bright stars in the dark night sky are ideally suited to this technique.

Photographs of everyday objects can be analyzed with blink comparison techniques; however, matching the common features of the two images requires similar image scale and perspective. As long as the two images are sufficiently close, the blink comparison can be carried out with an analysis program that fits one image to the other using standard data fitting techniques. The Change Detection System (CDS) from Idaho National 
Environmental Engineering Laboratory is a software package that distorts pairs of digital images to match common features. [2] After fitting, the two images are blink compared. The great advantage of CDS is that it enables blink comparison of photographs from hand-held cameras, as long as the scale and perspective of the two photographs are sufficiently similar. One current field application of CDS is the field inspection of cargo containers shipped from the United States to foreign sites, with photographs of the shipped and received container compared before acceptance of the shipment. [2]

Other tools: traditional film cameras still have a place, especially for those applications where maximum resolution is required. A Mamiya 645, a professional quality roll film camera, was often used in the initial stages of this project. There are other equivalent models from other vendors. Specialized lenses and light sources are often required for specialized photography. A zoom microscope with diffuse and axial illumination was invaluable for inspection of adhesive seals. The particular model was a Thales Optem Zoom Model 125.

The conclusion from this study is that the quality, availability, and simplicity of massmarket equipment allow a wide range of effective techniques to be demonstrated with simple techniques. Several different example scenarios were investigated.

\section{Example Applications and Scenarios}

This section reports evaluations of several representative applications, primarily involving seals and equipment inspection.

\subsection{Inspection of Tags and Seals}

Tags are unique identifiers or labels attached to an object; seals are devices to prevent and record unauthorized access to an object. Both are classed in general as tamper indicating devices (TID), even though only a seal is a TID. There are many different techniques, such as counterfeiting and replacement after removal, to subvert a TID. Two of the more powerful attack strategies are counterfeiting the seal and removing the seal with subsequent replacement. Counterfeiting the seal relies upon duplicating the seal and mounting it exactly within the precision of the inspection technique. Unique intrinsic features are powerful deterrents. Roger Johnston has produced several excellent papers on TID security as well as a comprehensive taxonomy of attacks. [3]

Defeating the counterfeiting attack relies upon making a tag or seal unique. The reflective particle tag (RPT), for example, consists of reflective chips in clear matrix. Flakes of glitter in clear epoxy forms an effective RPT. The RPT is considered highly resistant to counterfeiting since the reflective flakes are randomly oriented in the matrix and form a characteristic pattern depending upon viewing angle and illumination. While no tag or seal is absolutely proof against tampering, a tag or seal with a highly random 
pattern deters attack due to the perceived complexity and cost of producing a sufficiently similar counterfeit.

Photographic inspection techniques based upon blink comparison are highly effective at characterizing differences that occur to an object between photographs. Even if there are thousands of random objects in the photograph, a good blink comparison technique will align all the similar features sufficiently well that only the changes are apparent. This technique is highly effective with a sufficiently large random pattern. As a test case, photographic inspection has been applied to inspect a particular adhesive seal.

\subsubsection{A Study of 3M Confirm ${ }^{\mathrm{TM}}$ Tape}

Adhesive seals, also known as tamper tapes or pressure sensitive seals, are commonly used in safeguards applications. Photographic inspection of adhesive seals is a useful deterrent to tampering, since photography allows

- Documentation of intrinsic unique features of the seal, reducing the likelihood of successful counterfeiting.

- Inspecting and recording of the seal at high resolution as an archival record.

Unique features of the seal may include fine details of ink flow, patterns of embedded beads, or details of an applied feature such as a signature. Many of these features have been investigated in a study of Confirm ${ }^{\mathrm{TM}}$ tamper tape, a commercial product from $3 \mathrm{M}$ Corporation. [4] Confirm ${ }^{\mathrm{TM}}$ tape is composed of a layer of glass beads, embedded in a layer of adhesive. [5] Patterns and logos are formed in the bead layer by selective patterning of a reflecting layer, forming features seen only in retro-reflected light. The underlying substrate can be photographed through the tape, indexing the tape and its patterns to the surface of the secured item. An additional security feature is that the tape is frangible, making it difficult to remove and replace the tape without detection.

\section{ENLARGED SIDE VIEW}

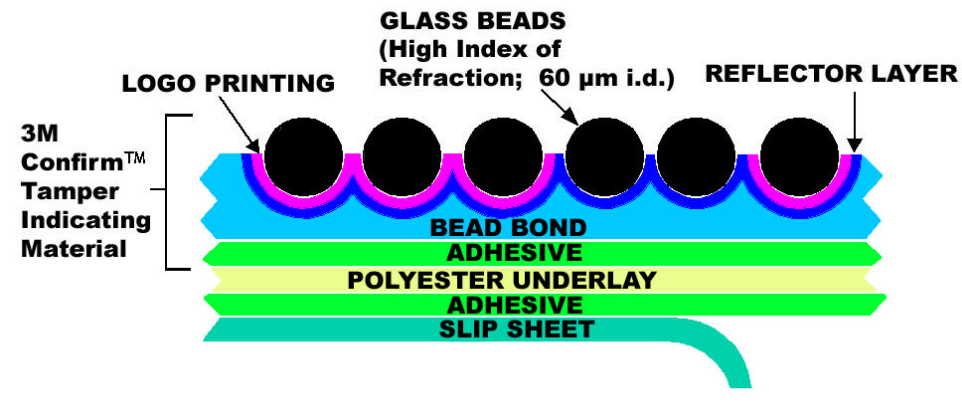

Figure 1: Schematic diagram of Confirm ${ }^{\mathrm{TM}}$ tamper tape, as manufactured for PNNL in 1994 


\subsubsection{Inspection of Confirm ${ }^{\mathrm{TM}}$ Tape with Hand Held Cameras}

Photographic inspection of tamper indicating devices can be a significant deterrent to attacks against a TID. In the case of a signed Confirm ${ }^{\mathrm{TM}}$ tape, any counterfeiting strategy must duplicate the flow of the ink around the beads. Simply photocopying the seal is not sufficient; comparing large area photography, with and without flash, will distinguish a true Confirm ${ }^{\mathrm{TM}}$ tape by the presence or absence of the retro-reflective pattern illustrated in Figure 2. Removal and replacement attacks are deterred since the bead pattern of the TID must not be disturbed. In a practical sense, these defenses are both enabled by change detection: the required comparison is made much easier by computer alignment of the similar features of the image.

Photographic comparison of Confirm $^{\mathrm{TM}}$ tape is illustrated below. Figure 2 is a photograph of a tape applied to an aluminum plate. The snowflake pattern is formed by the camera's flash illuminating the patterned retro-reflecting beads; this pattern is much fainter in diffuse ambient light. The location and date of application are written in the highly frangible central window while the signature of the applier is written across both the central window and the underlying polyester backing. The date and signature form intrinsic identifying features since the ink flows around the $60 \mu \mathrm{m}$ diameter beads in a highly irregular and random pattern.

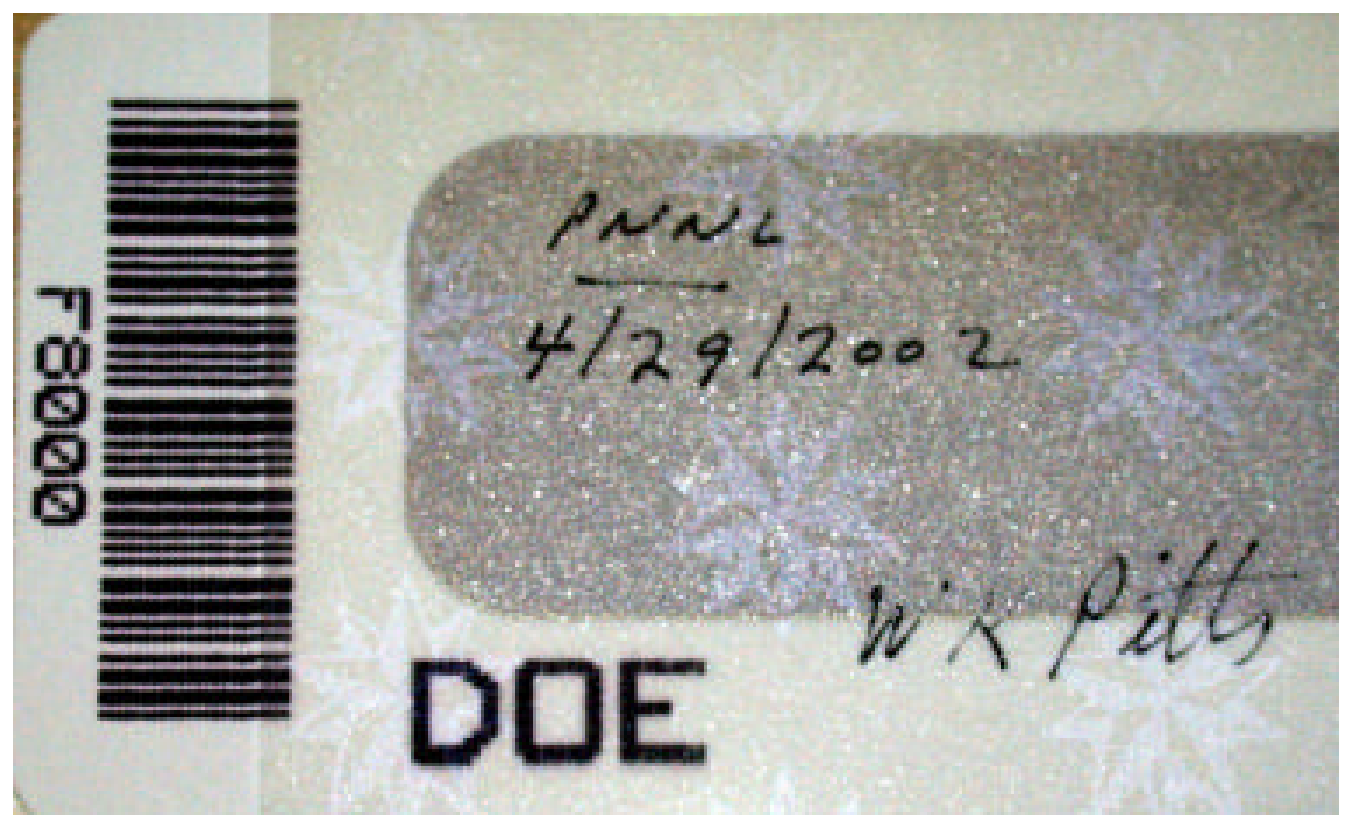

Figure 2: Illustrative photograph of an applied Confirm ${ }^{\mathrm{TM}}$ tape, showing the written data and the reflective pattern.

Figure 3 shows these features in more detail. The signed "PNNL" was photographed with a Nikon Coolpix 995 in extreme close-up mode. There is sufficient resolution to 
show the fine detail of ink flow around the top of the "P"; note that individual beads are easily distinguished, including strings of beads with preferential ink flow (top of Figure $3 b)$. This type of photograph is easily analyzed with CDS or other blink comparison system, since blink comparison emphasizes changes against a fixed background.

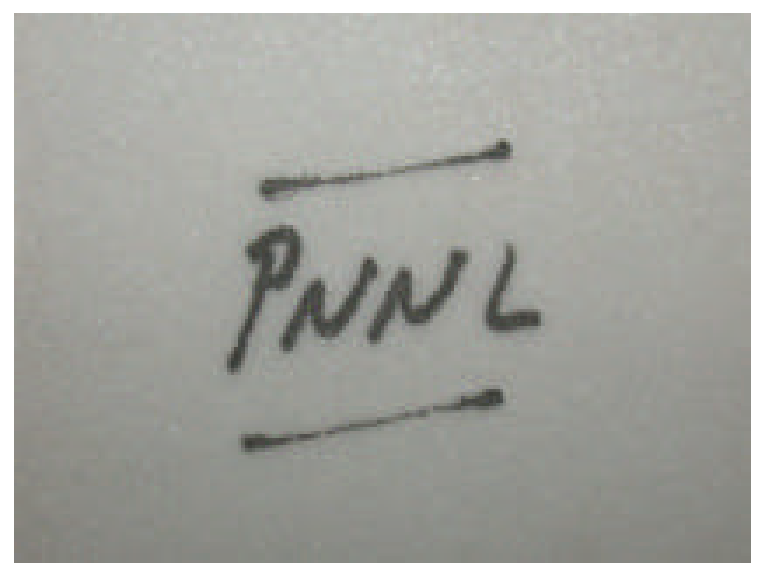

(3a)

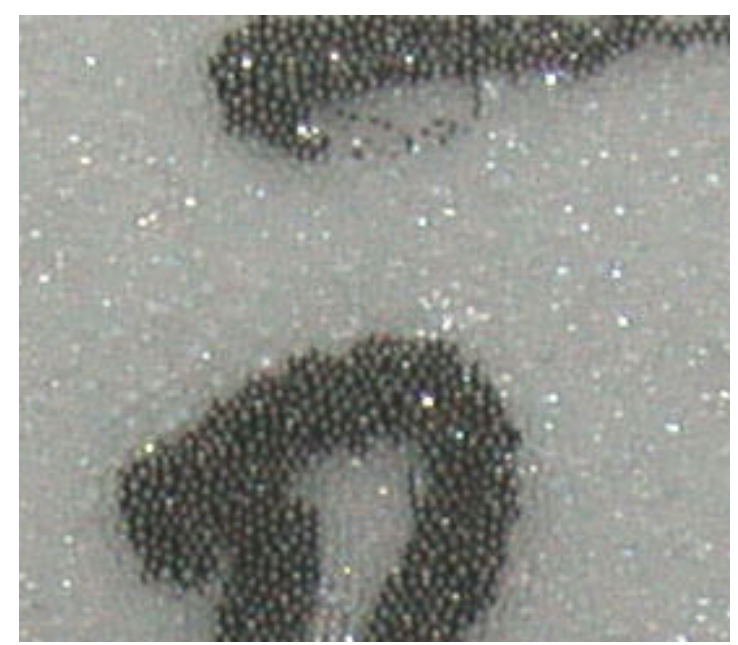

(3b)

Figure 3: Intrinsic feature in Confirm ${ }^{\mathrm{TM}}$ tape due to ink flow around the embedded glass beads. A signed feature was photographed at maximum close-up (Figure 4a), with a total object area of $2.2 \times$ $1.6 \mathrm{~cm}$. There is sufficient resolution to view the flow of ink around individual beads at the top of the "P" (Figure 3b). Ink flow around the beads is a characteristic feature of each signature.

\subsubsection{Inspection of Confirm ${ }^{\mathrm{TM}}$ with a Zoom Microscope}

While a hand held camera allows inspection of many features of Confirm ${ }^{\mathrm{TM}}$ tape, inspecting Confirm ${ }^{\mathrm{TM}}$ tape with a zoom microscope allows more detailed inspection:

- A zoom microscope allows detailed examination of the beads themselves, their colors, and their relative spacing

- Both diffuse and through-lens illumination are options, turning the retro reflection on or off.

The particular microscope was a Thales Optem Zoom 125 with both illumination options. Photographs of Confirm ${ }^{\mathrm{TM}}$ tape show that the ability to resolve details of ink flow in diffuse light and details of the beads in retro-reflected light is a very powerful technique, at the price of requiring a suitable microscope and illumination system. 


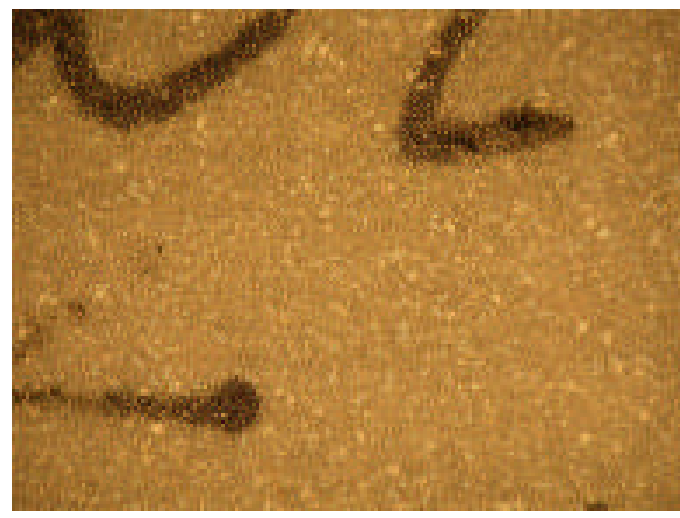

(4a)

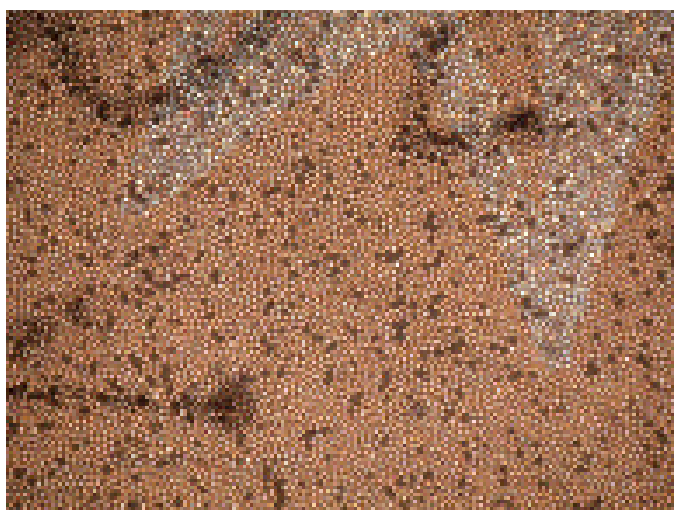

(4b)

Figure 4: Comparison of Confirm tape in diffuse (4a) and retroreflective (4b) mode. Note that the random ink flow shows up more clearly in diffuse lighting, while the random beak pattern shows up more clearly in retroreflective mode.

\subsubsection{An Example Implementation of an Inspection System}

A particular implementation of an inspection camera system is shown in Figure 5. This particular configuration is designed to photograph surface features at high-resolution using a camera mounted close to the floor while simultaneously photographing the surrounding area with a high camera and wide angle lens. A key feature is that most control functions (focus, flash, and field of view) are either automatic or easily verified by an observer, an important consideration in an inspection regime.

Two Nikon Coolpix 995 cameras are mounted on a framework, with one camera mounted close to the floor and the other mounted near waist level. Each camera is operated using a remote control pad mounted on the grip; the display from each camera is copied to a LCD monitor mounted near the grip. Even though these cameras auto-focus on the object, returning a positive green LED indicator on the camera when in focus, copying the display from the camera to the monitor allows the operator to independently view and verify the visual image and camera focus indicator on the LCD monitor. During use, the operator would position the camera mount, depress each camera control to initiate the auto-focus process, verify the focus after the green auto-focus LED is lit, verify that the flash unit LED indicators are on, and make the photograph. There is no need for the operator to kneel to the floor, and each operator action is easily verified. 


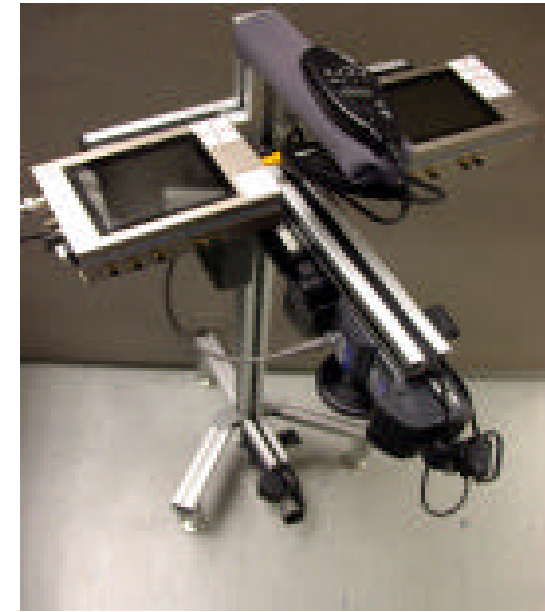

(5a)

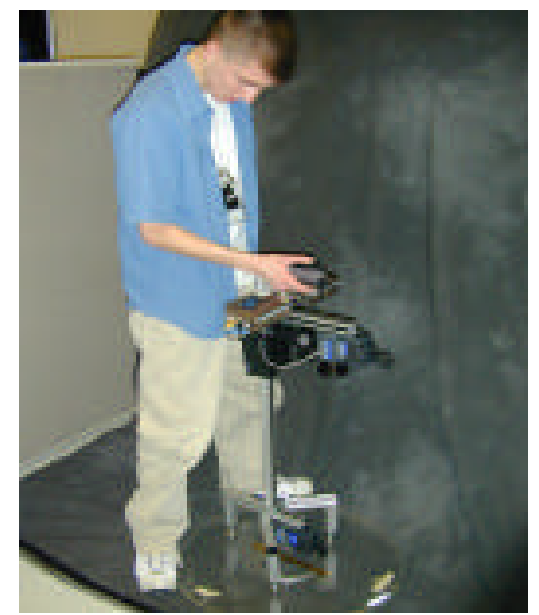

(5b)

Figure 5: An example implementation of a photographic inspection system. Figure 5a shows two cameras mounted on a jig, with a low-mounted camera for close up photography of features on a floor and a high mounted camera for simultaneous photography of the surrounding area. The LCD screens of the cameras are copied to separate external monitors for inspection of camera focus. Each camera is operated using touchpads mounted on the carrying handle. Figure $5 \mathrm{~b}$ shows the system in use on a plate.

Example photographs are shown below. Note that the high camera shows the overall condition of the seal and illuminates the retro-reflective security features of the Confirm $^{\mathrm{TM}}$ tape. One photograph is not sufficient to resolve the unique features illustrated in Figure 3, however, since the seal is only a small fraction of the available 3 megapixels of the camera. The low camera, however, will resolve the fine details of the signed data, as shown in Figure 7.

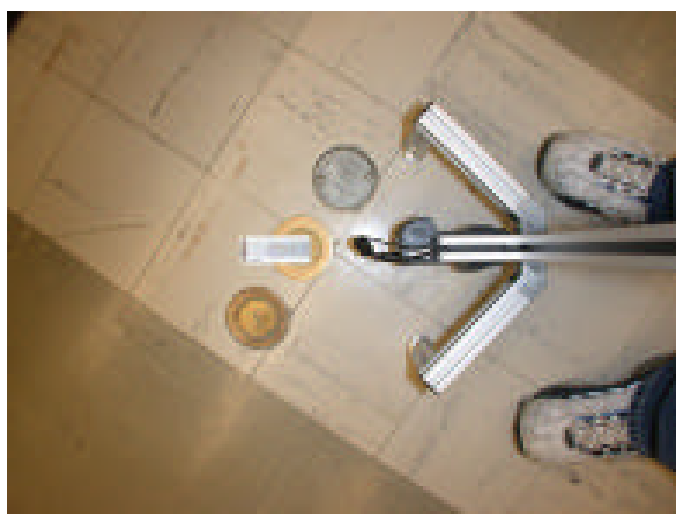

(6a)

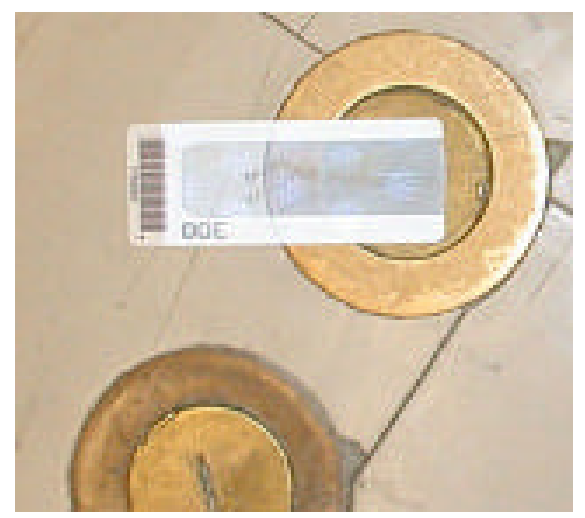

(6b)

Figure 6: Example photographs of floor level seals. Figure 6a shows the high camera view of the seal and the surrounding area. Figure $6 \mathrm{~b}$ shows an enlargement of the seal in Figure 6a, showing the retro-reflective security feature in the Confirm tape. Note the resolution loss upon enlargement; acquiring detail similar to that shown in Figure 4 requires the low camera. 


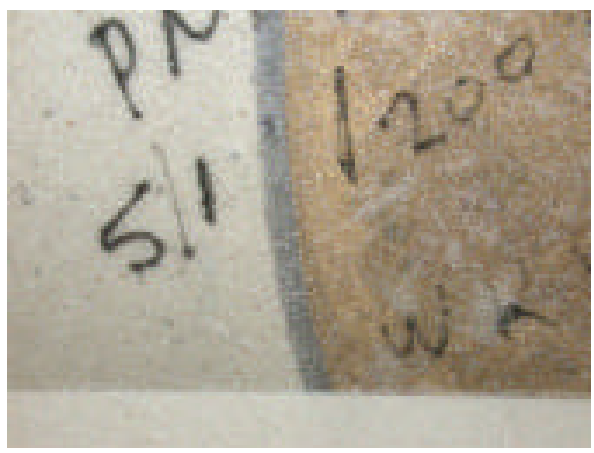

(7a)

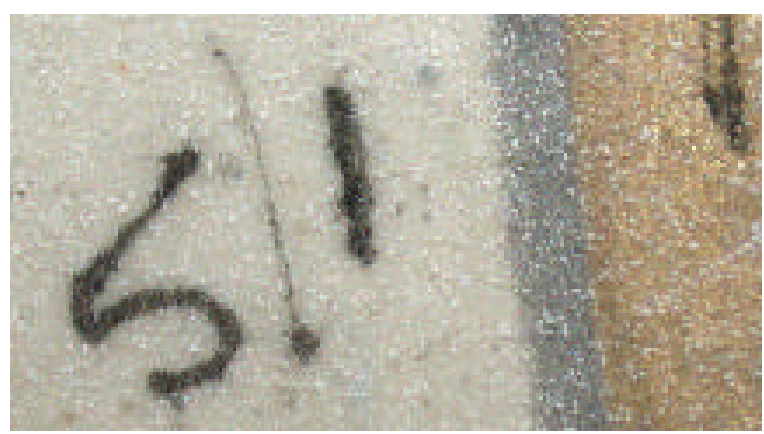

(7b)

Figure 7: Low camera views, full image (7a) and enlarged portion (7b). The characteristic features include the shape of the "5", it's distance to the edge of the flange, characteristic shape of the "w", and distance between the "5/1" and the "w".

\subsection{Inspection of Equipment}

\subsubsection{Introduction and Test Case}

Equipment inspection often requires that changes to the equipment from an earlier inspection be detected. The ability to track a change is a powerful deterrent to a change. An example application is the inspection of electronic assemblies such as circuit boards.

Inspection of Intrinsic Features in Electronic Assemblies: The assembly of electronic circuit boards consists of forming conducting metal traces on the surface of the board, placing electronic components in the proper location, soldering components, cleaning the board, and possibly applying a protective conformal overcoat. Characteristic features are formed during each step. Metal traces produced by photolithography may be offset to mechanical features produced by drilling the board for connectors. Required mechanical tolerances tend to produce a degree of randomness in component placement. Solder flows are controlled by local cooling and fluid flow, tending to produce different shapes at each soldered connection. Replacing a component can lead to localized heating of the underlying circuit board, producing a stress pattern. Discrete components such as capacitors, inductors, and large resistors may have distinguishable changes in orientation.

The photographs below show the substitution of an electronic component. The chip resistor in the upper left was removed and replaced. Before and after photographs show that the orientation of the resistor has changed with respect to the other components and that the solder bumps have been changed. In a still photograph, the difference is easily seen once identified. Blink comparison, after alignment to the unmodified features, easily shows the replacement. A further advantage is that larger images can be compared with blink comparison, as opposed to detailed visual inspection. The images in Figure 1 
have been isolated for this figure; the original image was approximately 25 times larger in area and the changes easily isolated with CDS.

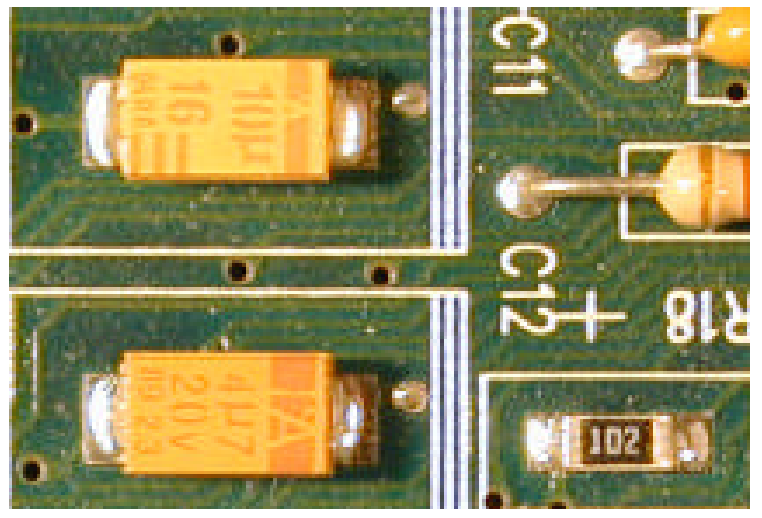

(8a)

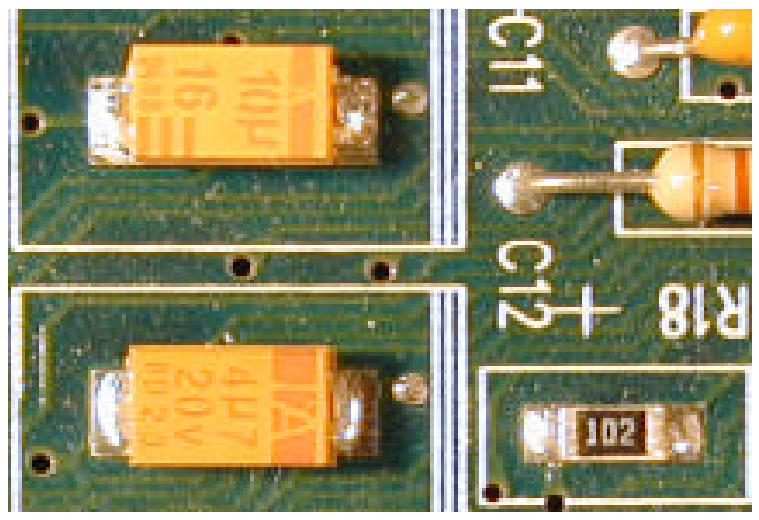

(8b)

Figure 8: Comparison of before (1a) and after (1b) photographs of a removed and replaced chip capacitor (upper left). Note that both the location and orientation of the capacitor have changed as well as the shape of the solder connections.

\subsubsection{Example Application to Modified Circuit Boards}

A before and after comparison was possible at a vendor where there were several documented circuit boards that required repair; the vendor made the photographs without direct involvement by PNNL personnel. These photographs are a realistic test of the utility of change detection applied to electronic components. There were several different types of changes that were noted as being characteristic of the repair process:

- Changes in cables and components: addition of cables, modifications of cables, and orientation of components

- Changes in shape of solder bumps: as solder is heated and cooled, the shape of the bump changes. Since the shape of the bump is determined by fluid dynamics of the molten solder, with an initial shape determined by how the soldering iron is pulled away from the board, the shape of the solder is difficult to control and is an intrinsic feature.

- Changes in board color due to heating: heating the board will change the color of the circuit board next to the connection.

Representative examples are shown below. In several cases the photograph color and contrast has been adjusted to better show the features. Note that these photographs are made using a standard camera (Mamiya 645) mounted on a jig; inspection with the zoom microscope system and better lighting control would reveal a further level of detail.

The figures below show a characteristic feature, apparently formed by flux wetting the board. 
PNNL-14174

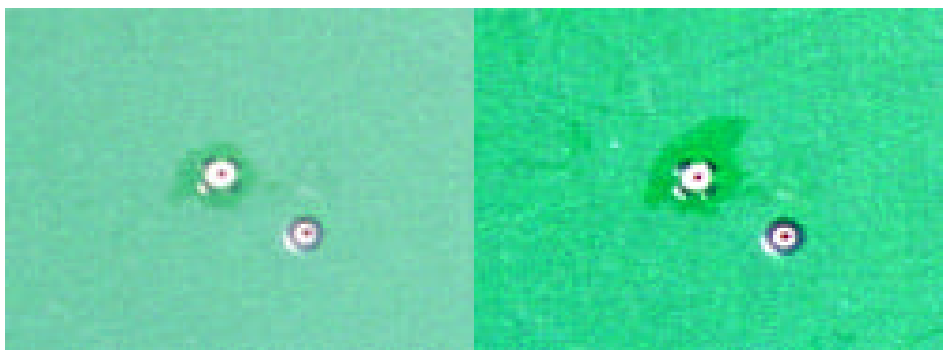

Original

Modified

Figure 9: Comparison of a solder point, showing a change in the flux stain.

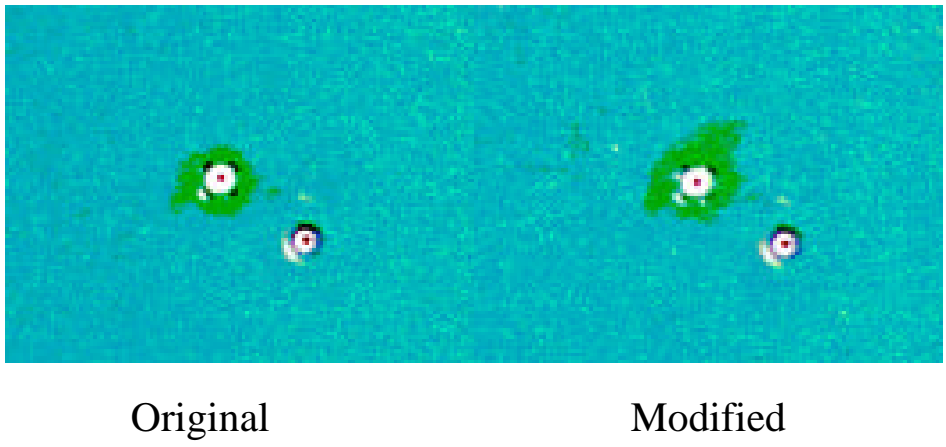

Figure 10: Comparison of the features shown in Figure 9, using color adjustment to heighten the contrast.

The figures below show an extreme case of thermal damage around the solder point in the lower left corner. In addition, note that the shape of the solder bump has changed. Shape changes in solder bumps are an intrinsic feature, formed by details of heat flow and cooling. Thus, any soldering done to an electronic board can be detected through photographic analysis. An interesting example of a true feature is shown in Figure 11, where a fingerprint has been enhanced by adjusting the color. 
PNNL-14174

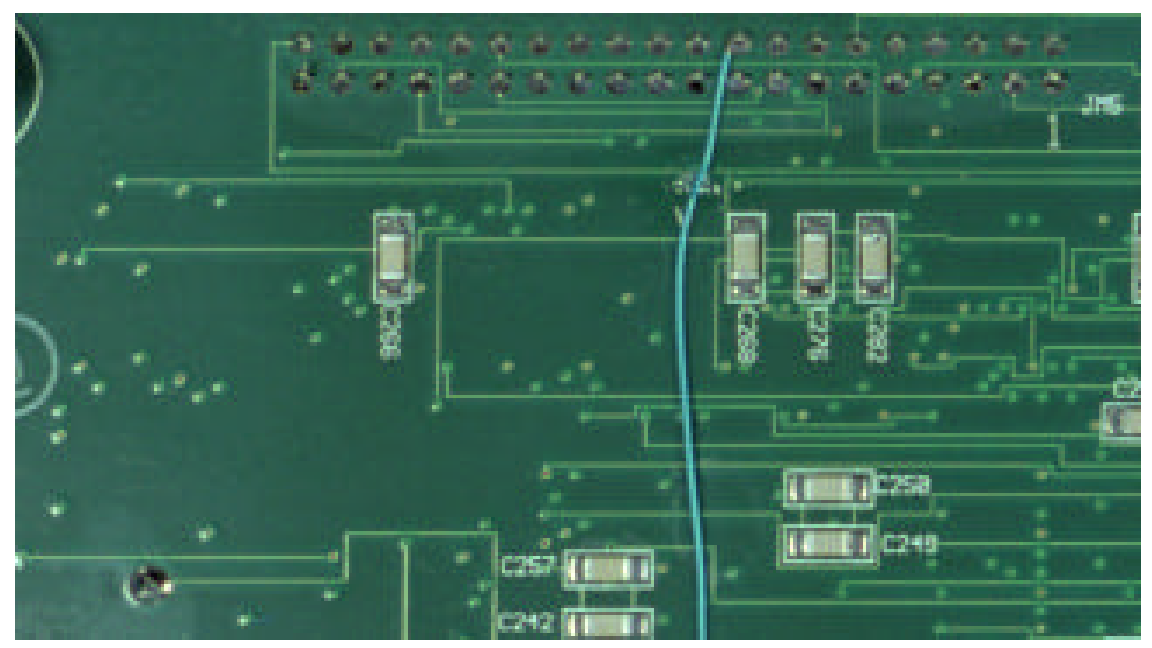

Original

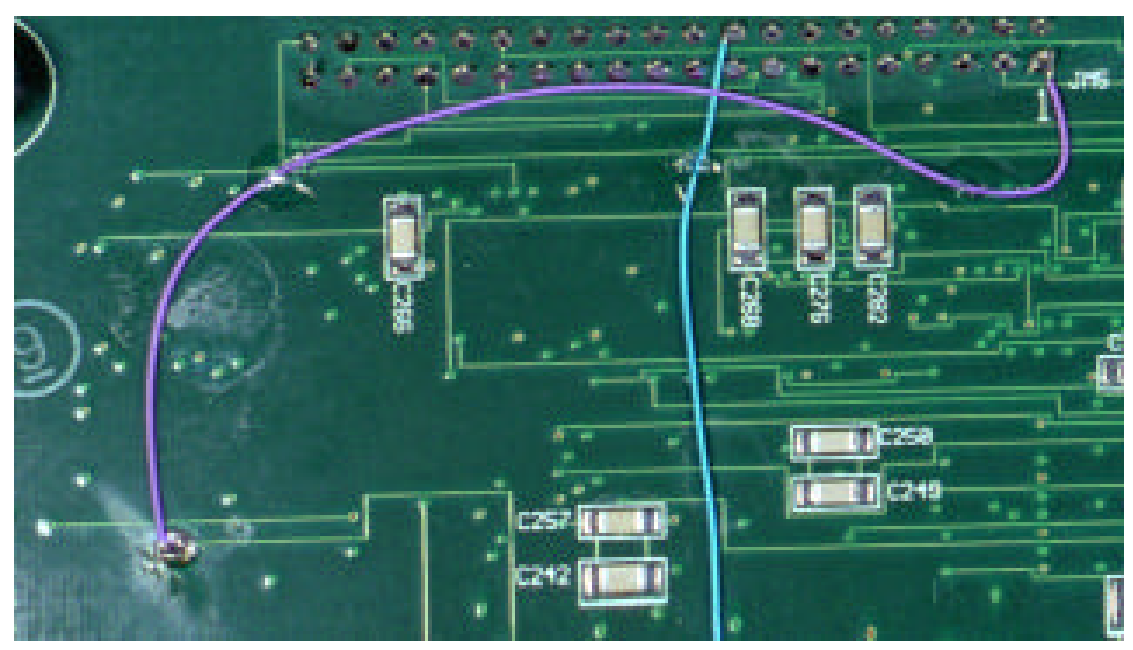

Modified

Figure 10: Example of changes in solder stains and points. 
PNNL-14174

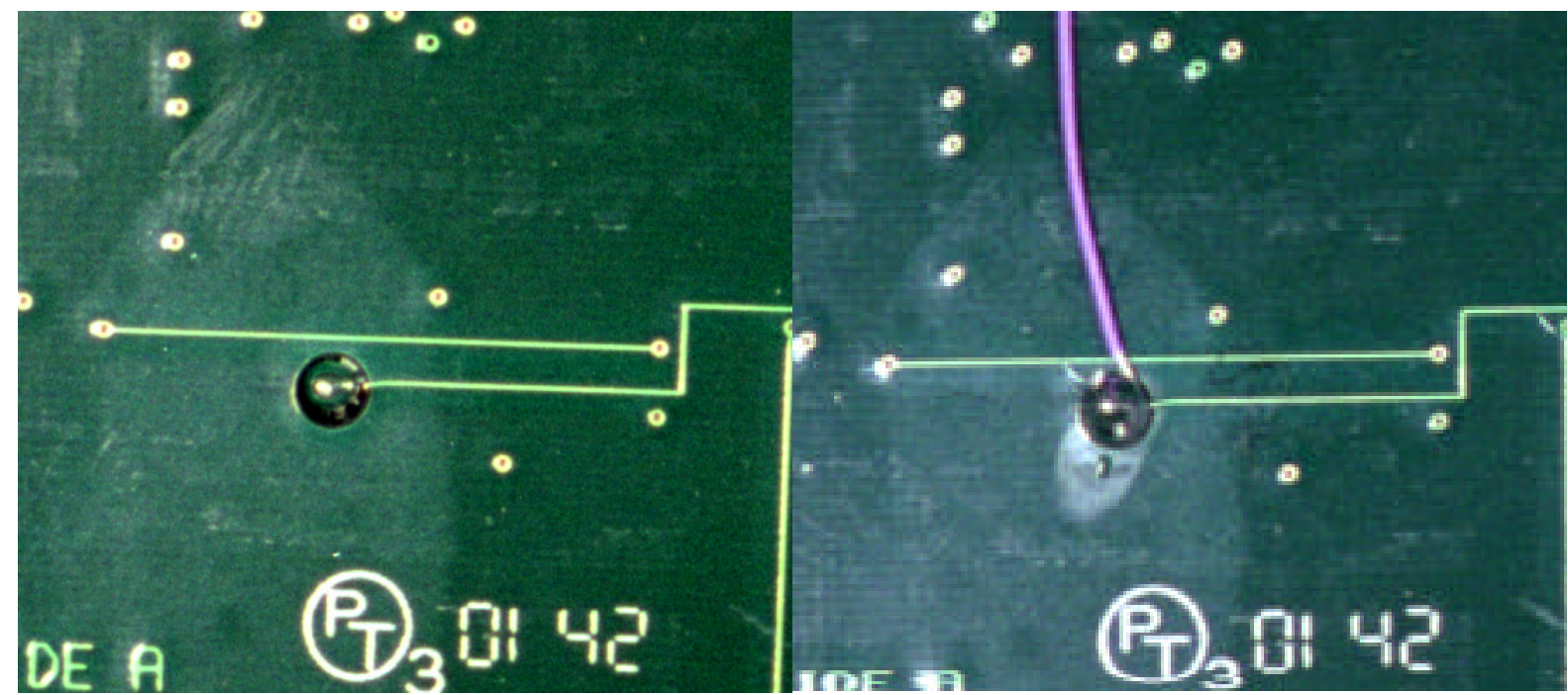

Original

Modified

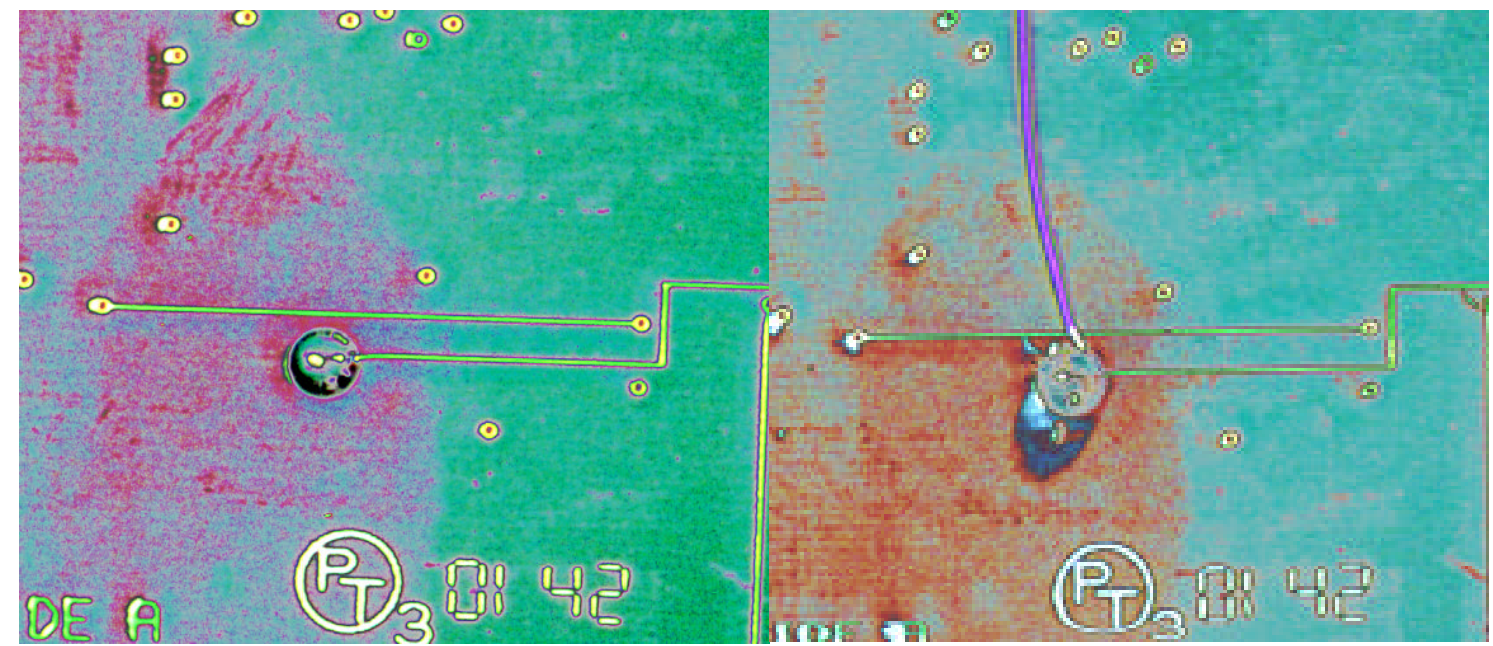

Original

Modified

Figure 11: Example of further changes during addition of a cable. Note fingerprint in upper left corner, emphasized in lower set of photographs with adjusted color change. 


\subsubsection{Inspection of Enclosures}

Inspection of enclosures would be expected to rely upon the techniques shown above for seals and equipment. Using intrinsic features in photographs, photographic analysis is possible allowing for the discovery of changes made to a system.

\subsection{Scenarios}

Some example scenarios are illustrated below for the purposes of illustrated required technical development. Using the standard phrasing, "Monitor" refers to the party that is examining the facility of the "Host". In all cases, there is an expectation that the Host

- Has some degree of inspection concerning photographs acquired by the Monitor, ranging from being furnished a second copy of the photograph or media to other extreme of furnishing photographs to the Monitor.

- Furnishes all photographic equipment and media that will be used for photographic documentation.

Scenarios with complete Monitor control, including the use of Monitor's equipment and no information release to the Host, have not been considered.

\subsubsection{Photography with Monitor Control and Original Media Removal}

In this scenario the expectation is that the Host furnishes a selection of identical cameras and media to the Monitor, who selects a camera and associated media using Random Selection guidelines. ${ }^{1}$ This scenario further assumes that the Monitor may make the photograph, using equipment furnished by the Host. The Monitor may bring in a color chart and a resolution chart to verify camera operation. Photographs are available on original media (either digital memory or analog film such as Polaroid ${ }^{\mathrm{TM}}$ ) and removed by the Monitor. A copy is furnished to the Host for evaluation.

The required technical development in this scenario is development of procedures for photographic inspection, optimized for minimal time and presence, and camera technical performance. For example, efficient photography might require mounting jigs such as those used to photograph electronic components and floor seals.

\footnotetext{
${ }^{1}$ Random Selection is an Authentication tool to build confidence in measurement equipment. Two or more identical items are presented by the Host to the Monitor, with each item being immediately ready for measurements. The Monitor selects one item and retains it in custody for later private inspection and selects the other item for making measurements. Its Authentication value is that the Host knows that the Monitor can analyze the removed item for performance and integrity in subsequent private tests, verifying that the instrument is as it should be.
} 


\subsubsection{Photography With Host Approval and No Original Media Removal}

In this scenario Host furnishes a selection of identical cameras and media to the Monitor, who selects a camera and associated media using Random Selection guidelines. Host personnel make the photograph using equipment furnished by the Host, under the direct supervision of the Monitor. The Monitor may bring in a color chart and a resolution target to verify camera operation. Photographs are available on original media (either digital memory or analog film such as Polaroid ${ }^{\mathrm{TM}}$ ) but may not be removed by the Monitor as original media. It is necessary to make a copy of the media before removal from the site.

The required technical developments in this scenario, beyond that listed in 2.4.1, are

(1) Specification of procedures in which the Monitor can observe the camera settings and image when the Host is producing the photograph,

(2) Specification of mutually acceptable procedures for removing the photograph.

Item (1) may be accomplished by using digital cameras with auto focus and other automatic settings that are displayed on an auxiliary monitor. These procedures were demonstrated in the prototype system for inspecting floor seals. Item (2) may be accomplished by either printing a copy directly from the camera to a printer, scanning and printing the photograph, or furnishing a duplicate copy of the electronic media. In this case, it is necessary to verify that the image has not been changed or modified. If the media is a digital memory device like a CompactFlash ${ }^{\mathrm{TM}}$ card, the best technique would be to directly compare the two versions using data authentication and verification techniques such as direct comparison or hash function techniques. Rather than develop complex techniques requiring sophisticated equipment, this case study investigated techniques for duplicating photographs using commercially available mass market printers and scanners.

\subsubsection{Photography Supplied by the Host from Archival Photographs}

This scenario assumes that the Host flatly prohibits photography in the Host facility, but will instead furnish photographs from an archive. This undesirable scenario requires that there be some comparison of the image to the item it purports to represent. In this case, it would be necessary to physically compare selected photographs or features of photographs to the item before accepting the photograph as genuine. It is critical that the Monitor select the particular feature to be inspected.

Since the Host would be supplying the photographs, there is no required technology development. There is a need to specify the Monitor's access to the item and any ancillary inspection equipment such as magnifying lens or zoom microscopes. 


\section{Detailed Technical Results}

This case study concentrated upon several different systems, concentrating primarily upon consumer mass-market equipment. The following are our recommendations for equipment that is well suited for photographic analysis.

\subsection{Cameras and Optical Systems}

\subsubsection{The Nikon Coolpix 995 Digital Camera}

The Nikon Coolpix 995 is an easy-to-use digital camera that was the upper end of the Nikon consumer mass-market product line during most of this project. [6] Many other vendors vend equivalent models. It allows the user to quickly take and view a photograph. The Nikon uses flash memory cards, which can be used to directly download pictures to a computer or printer. A single FlashCard ${ }^{\mathrm{TM}}$ memory unit can hold hundreds of digital pictures at acceptable quality. In comparison, one roll of film in the Mamiya 645 can hold merely thirty pictures. (Mamiya, 645 3.1.2)

- The Nikon is best suited for any situation where time is limited and the picture needs to be immediately viewed.

- Copying the display to a small monitor allows verification of the camera settings and focus.

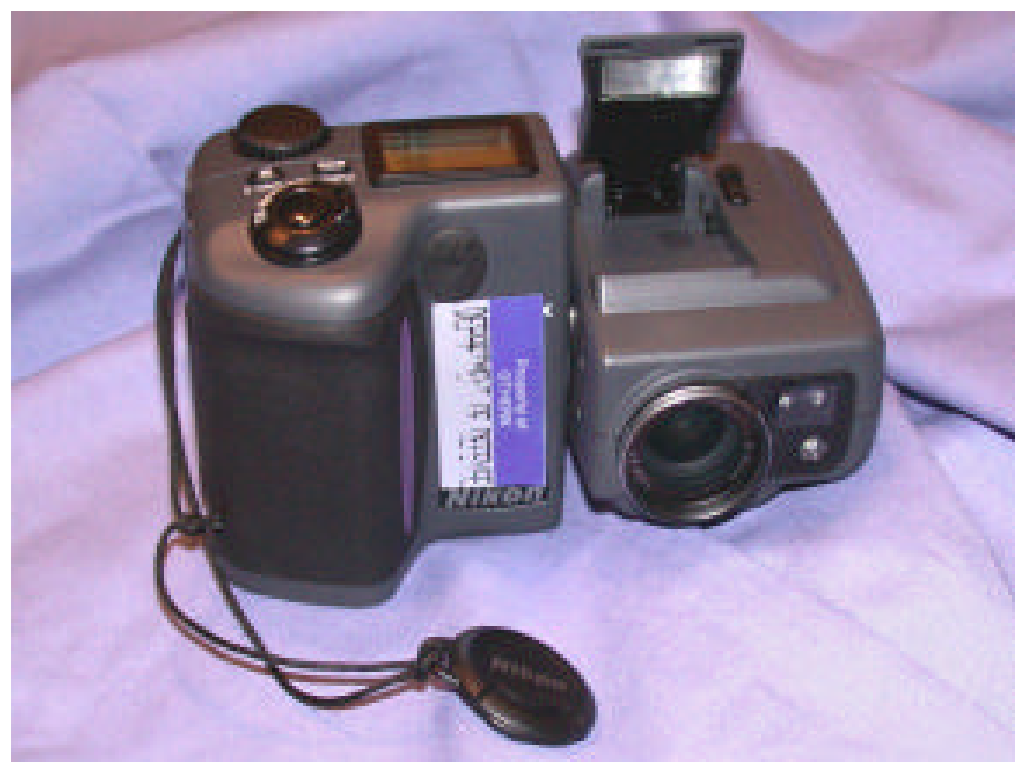

Figure 12: The Nikon Coolpix 995 camera, a mass-market digital camera with good macro photography capability. 
PNNL-14174

\section{Optimum Settings:}

(Best Close-Up)

Lens to subject distance: $1.5-6.0 \mathrm{~cm}$

Focus: Tulip- Close-up mode (where tulip turns yellow)

Zoom: Half-Full

F-Stop: 4.0-11.0

Mode dial: Manual exposure

Manual priority: Aperture

Photo quality: Fine

Internal flash: On, flipped up

Flash setting: Flash only

White balance: Auto

Metering: Spot

Best-shot selector: Off

Image adjustment: Auto

Saturation control: Normal

Lens: Normal

Focus: AF area mode

Image sharpening: Auto

Auto bracketing: Off

Noise reduction: Off

\section{Results:}

Magnification: 8x-15x

Resolution:

Hard Copy- 35.9 cycles per mm

Computer File- 38.0 cycles per mm

Note: Table 1 shows results received when the Nikon Coolpix 995 is used with alternate settings, and in particular, at longer working distances.

\section{Discussion:}

\section{Advantages:}

- Lightweight

- Compact

- Mobile

- Automatic exposure setting for quick and simple pictures

- Manual exposure, aperture, shutter speed, and focusing settings

- Immediate results 
- Flash Memory Cards download to computer quickly and with highest picture accuracy

- Mounts stands and tripods without difficulty

- Multiple lens attachments

- External flash connection

- Built-in pop-up flash

\section{Disadvantages:}

- Using the flash with a lens attachment requires an external speed light or other flash device.

- Short battery life.

- Internal flash provides only a small circle of light in close-up pictures.

\section{Operation Guidelines}

- Turn the Mode dial to M (choose A if inexperienced user must take quick photo).

- Set photo quality to Fine, focus to Tulip (Press the M-Focus button until a tulip appears in upper right hand corner of screen), and manual priority to Aperture.

- If a special lens or external flash is used, ensure that the camera is set to the correct settings.

- Zoom in on the subject using the W/T buttons.

- Use the scroll dial to move F-stop to highest available value.

- To focus, lightly press the shutter release button and wait for the camera to focus in on the subject (tulip in upper right corner must be yellow to focus correctly).

- Finally, fully press the shutter release button to take the photograph.

- The photograph will appear in the LCD view screen for a brief period of time. If the picture is focused correctly and has the proper lighting wait a few seconds until the picture disappears. The picture is now loaded on to the flash card.

- To view the picture again, turn the mode dial to the play mode and use the multiselector to scan through the pictures.

- To replace the battery: open compartment, remove battery, and insert replacement battery.

The following table shows the measured resolution of the Nikon Coolpix 995 camera under different settings and lenses using a glass USAF 1951 resolution target. Any instance in which basic or normal qualities did not differ in resolution from fine and high qualities the resolution was recorded only as fine and high quality. 


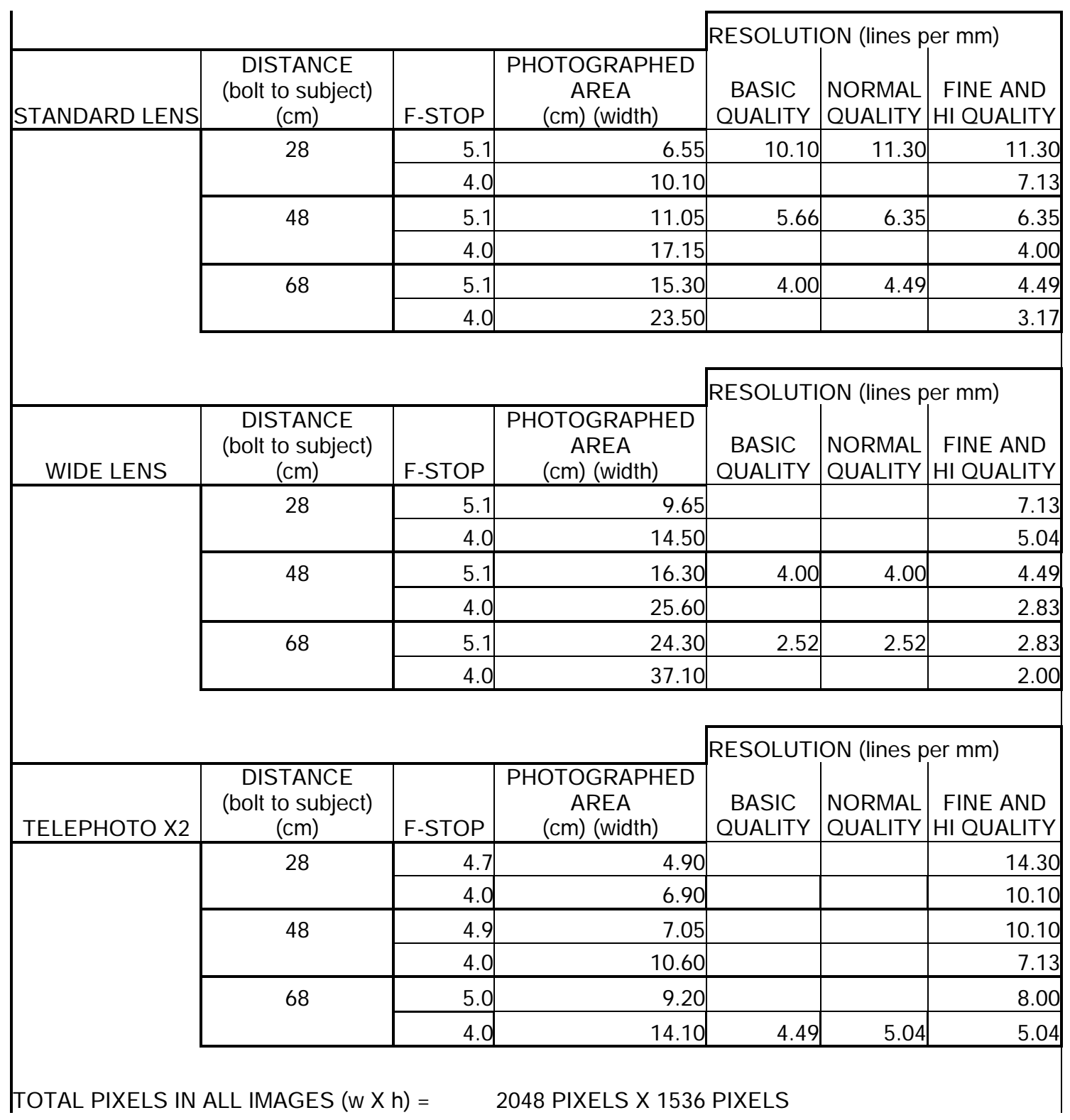

Table 1: Demonstration resolution test of the Nikon Coolpix 995. Note that the working distance was chosen for a convenient demonstration of the technique with both the telephoto and wide angles lens as well as no auxiliary lens.

As seen in the table above, resolution varies with your choice of lens and quality. A telephoto lens will yield the best resolution, while a wide angle lens yields the worst. The best resolution to take pictures at is fine quality, since it has the same resolution as high quality but takes up less memory space. 


\subsubsection{The Mamiya 645 Professional Roll Film Camera}

The Mamiya 645 is a medium format camera with the capability of using both instant and 120 film. This camera feature ensures reliable close-up photos. A hard copy of the photo can be produced instantly, valuable for verifying correct photo settings and leaving the photo site with a physical means for photograph inspection. A film roll can be developed for photos with more detailed features and higher resolution. The camera includes numerous manual settings, including depth of field and shutter speed. The flash on the camera produces a consistent photo when used with Automatic shutter speed.

- With the macro zoom lens, the Mamiya 645 provides the highest resolution over the largest area of any of the cameras tested and should be utilized on a tripod or stand photographing aspects located between $14 \mathrm{~cm}$ and $500 \mathrm{~cm}$ from the $120 \mathrm{~mm}$ camera lens.

- Developing and scanning analog film can become a significant logistical constraint.

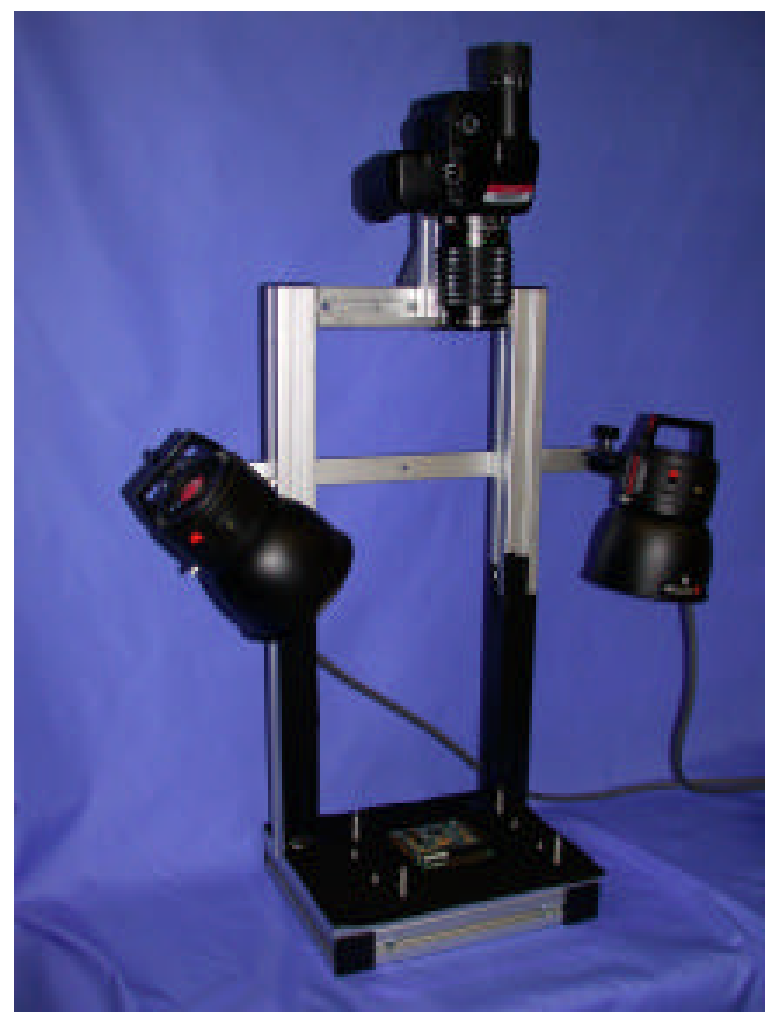

Figure 13: The Mamiya 645 mounted on a copy stand for circuit board photography.

\section{Optimum Settings}

(Best Close-Up)

End of lens to subject distance: $14.6 \mathrm{~cm}$

Magnification: 1x, full 
F-stop: 32

Shutter speed: Automatic

\section{Results}

Magnification: $1.1 \mathrm{x}$

Resolution:

Hard Copy- 14.3 cycles per mm

Computer File- 13.5 cycles per mm

\section{Discussion}

Advantages:

- Manual mode checks depth of field before taking photo (yellow light appears in viewfinder)

- Polaroid and film backs allow for both an immediate hard copy photo and a more distinct developed photo

- Fits most tripods

Disadvantages:

- Image loses clarity in transition from actual photograph to computer analysis

- 120 film is more difficult and costly to develop than other film types

- Bulky Polaroid back makes tripod usage more challenging

\section{Operation Guidelines}

- Set the F-stop by turning the aperture ring until the correct value is aligned with the red dot.

- Set the shutter speed by twisting the shutter speed dial until the white index line is aligned with $\mathrm{A}$.

- Focus the camera by rotating the front most section of the lens until the two halves of the split-image circle combine into one.

- Verify that the settings are suitable with a test picture on the Polaroid film back (See below: Taking the Test Picture with Polaroid Film)

- Once each photo is taken the film will automatically advance.

\section{Taking the Test Picture with Polaroid Film}

- Remove film back and attach the Polaroid film holder using the buttons on the back of the camera.

- Pull the leader paper out of the Polaroid film holder until the first white paper tab comes out of the film holder.

- Press the shutter release button on top of the power drive grip.

- Pull out the white tab from the film holder.

- Pull out the gray paper tab connected to the photograph. 
- Allow the photo to sit for a minimum of 30 seconds.

- Examine photo quality, if appropriate settings were used, remove Polaroid film holder, reattach film back, and begin taking film pictures.

\subsubsection{The Polaroid MP5 Forensics Camera}

The Polaroid Macro 5 is an instant print close-up camera [8] It is lightweight and maneuverable, and of the cameras tested is the quickest and easiest to operate. Although the camera can take reliable photos at camera to subject distances of up to $125 \mathrm{~cm}$, it is ideal for closer subjects. The flash can reach in these closer set-ups and can provide repeatable lighting.

- The Polaroid Macro 5 is best suited for immediate close-up results of subjects requiring a mobile camera.

- There is an immediate comparison between the object and the photograph.

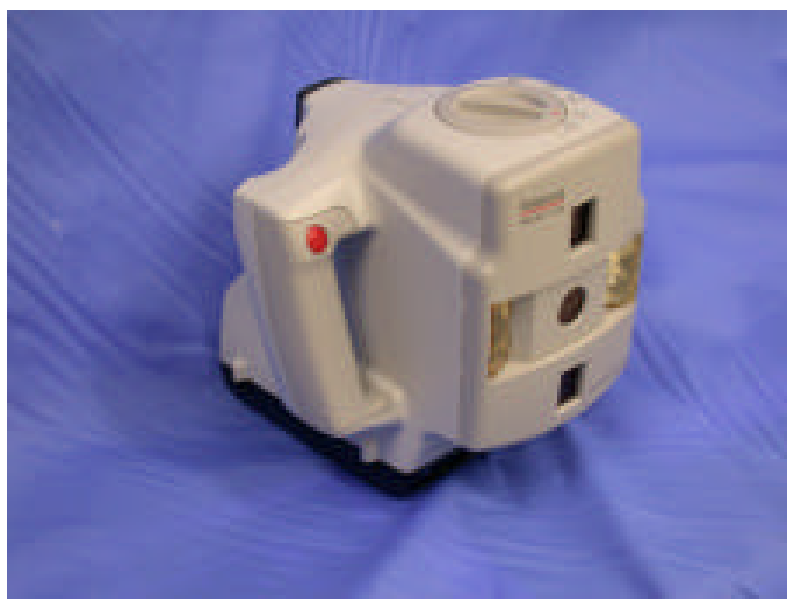

Figure 14: The Polaroid MP5, a professional camera with instant analog photographs.

\section{Optimum Settings}

(Best Close-Up)

Camera to subject distance: $8 \mathrm{~cm}$

Magnification: $3 \mathrm{x}$

Flash: Automatic

\section{Results:}

Magnification: $3 \mathrm{x}$ 
Resolution:

Hard Copy- 14.3 cycles per mm

Computer File- 12.7 cycles per $\mathrm{mm}$

\section{Discussion:}

Advantages:

- Mobile

- Simple to operate

- Consistently accurate lighting

- Immediate results

- Mounts tripods without difficulty

Disadvantages:

- Print loses some clarity when scanned to computer for comparison

\section{Operation Guidelines:}

- Press power button-flashes will charge, wait for green light.

- Turn magnification dial to $3 \mathrm{x}$ or desired setting.

\subsubsection{The Thales Zoom Microscope}

The Microscope System utilized for photographic detection is composed of multiple zoom optical couplers from Thales Optem Inc. [9] A dual capability light source from Dolan-Jenner Industries was paired with this system. When the light source was tested with $3 \mathrm{M}^{\mathrm{TM}}$ Confirm Tape, it provided ring light for surface bead viewing and back light for reflective pattern viewing. (3M $\mathrm{M}^{\mathrm{TM}}$ Confirm Tape 2.1.2) The system is used with an attached Nikon Coolpix 995 digital camera (3.3.1). This optical system possesses the highest magnification and best close-up capability of any of the other options.

- The microscope system is best suited for extreme close-up photos of movable subjects. It is ideal for pictures of $3 \mathrm{M}^{\mathrm{TM}}$ Confirm Tape due to the system's unique retro-reflective lighting capability. $\left(3 \mathrm{M}^{\mathrm{TM}}\right.$ Confirm Tape 2.1 .2$)$

- The microscope requires a camera; all tests used the Nikon Coolpix 995.

\section{Optimum Settings (best close-up):}

Ring light to subject distance: $6.0-7.0 \mathrm{~mm}$

Microscope zoom: 4.0-0.52

Nikon Coolpix 995 Optimum Settings (3.1.1) 


\section{Results:}

Magnification: 50x-100x

Resolution:

- Hard Copy- 60-110 cycles per mm

- Computer File- 60-120 cycles per mm

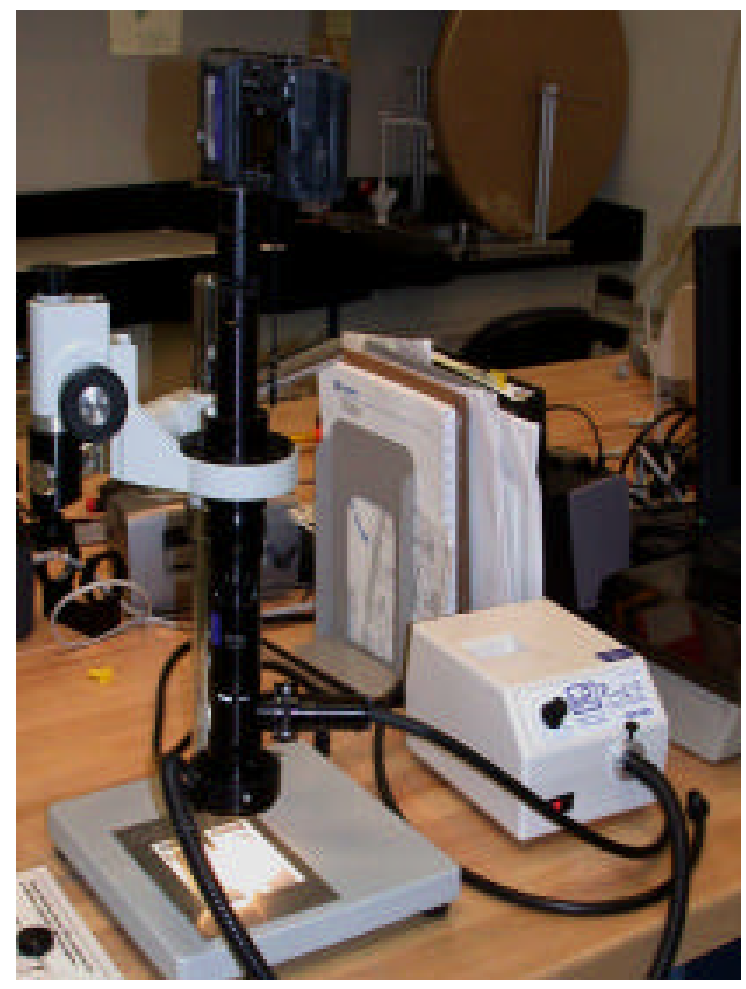

Figure 15: The Thales zoom microscope system and illuminator.

\section{Discussion:}

\section{Advantages:}

- Arm of system easily rotates

- Best magnification and resolution capability

- Simple to operate

- Two light source types (ring, retro-reflective)

- Quick camera attachment

\section{Disadvantages:}

- Difficult to move (very heavy base, awkward protruding arm)

- Limited use because subject must be moved to microscope, or the microscope must be mounted on a special jig 


\section{Operation Guidelines:}

- Adjust zoom using numbered ring and system height using knob.

- Nikon Coolpix 995 Operation Guidelines (3.1.1).

\subsubsection{A Demonstration System for Floor Seals}

The Cane Cam is a camera system that specializes in taking both close-up and wide area shots. Using two Nikon Coolpix 995 cameras, one Nikon Speed Light, and two LCD monitors, the Cane Cam is a tripod setup that allows for quick and consistently accurate photographs of any surface.

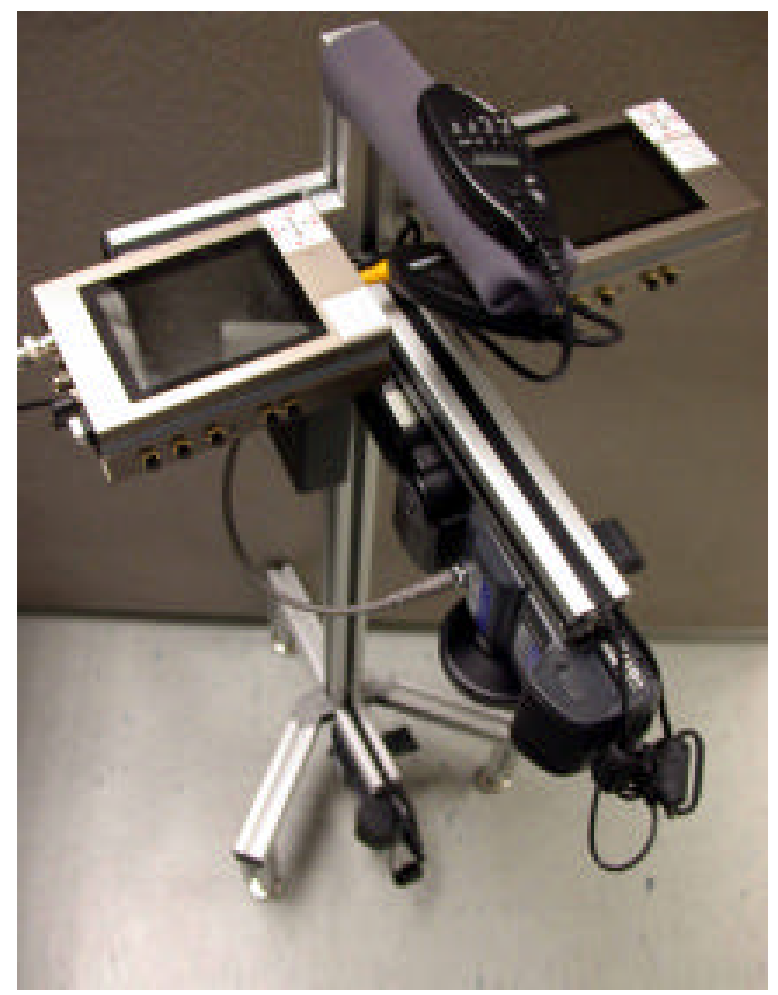

Figure 16: The Cane Cam system for photographing floor features. 

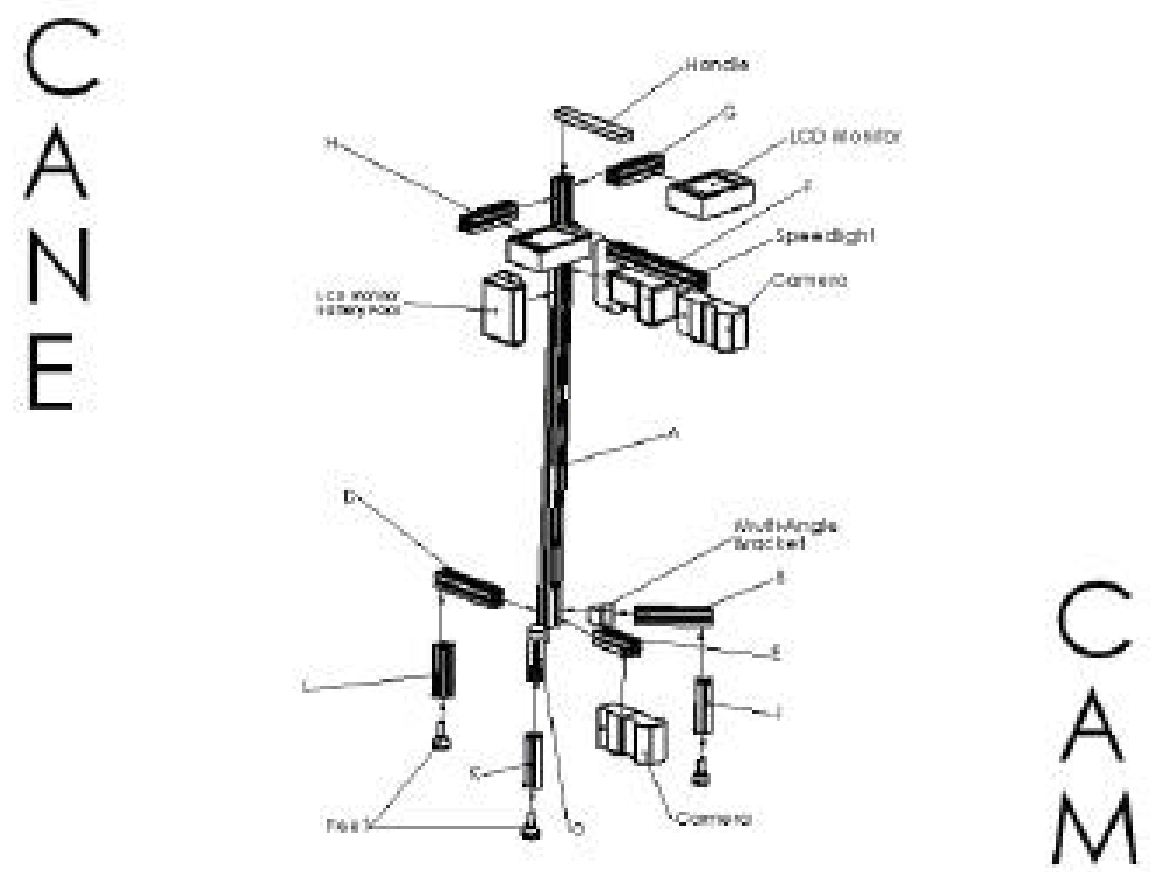

Figure 17: Assembly drawing for Cane Cam.

\section{Assembling the Cane Cam:}

\section{Materials}

- Nine 30 x 30mm Bosch pieces, labeled A through L:

- A: 3'

- B, C: 5.79"

- D: 6"

- E: $3.625 "$

- F: $9.4375 "$

- G, H: 4"

- I: 8 "

- J, K, and L: 4" plus attached feet

- Two $45^{\circ}$ angle Brackets, labeled M

- Two Bracket End Caps

- Eight Plastic Adapters (already in place)

- Three Bosch End Covers (already in place)

- $5 / 32$ socket head screws

- 3 metric truss head screws

- $\quad 3 / 16$ socket head screws

- Two Nikon Coolpix 995 Cameras

- Two LCD Monitors

- Four Battery Packs 
- Holding Handle

- 5/32 Allen Wrench

- 3 metric Allen Wrench

- 3/16 Allen Wrench

Note: To prepare a connection: slightly screw a T-nut on to the top few threads of one of the 3 metric screws. Position the underside of the screw head against the restraint on the end of the Bosch so the T-nut is protruding from the end of the Bosch. Refer to Diagram 1 .

1. Prepare a connection between $\mathrm{D}$ and $\mathrm{L}$. Insert the T-nuts of $\mathrm{L}$ into the well of $\mathrm{D}$ to form a $90^{\circ}$ angle between the two Bosch pieces. Black lines on D will guide where $\mathrm{L}$ attaches to $\mathrm{D}$. Make a firm connection by tightening the two 3 metric screws. This will cause each T-nut to rotate perpendicular to the inserted position and the end of each 3 metric screw to touch the back of the well.

2. Repeat for a connection between $\mathrm{C}$ and $\mathrm{K}$, and $\mathrm{B}$ and $\mathrm{J}$.

3. On Bosch B insert a 3/16 screw with a washer into the hole in the middle of the outward side of bracket M. Slightly screw a T-nut on to the end of the $3 / 16$ screw. Insert the T-nut in to the well of Bosch $\mathrm{A}$ at the point where the top of the bracket meets the black lines labeled 'B' and ' $C$ '. Refer to diagram 2.

4. Repeat step 3 for Bosch C.

5. Snap in the end caps for the $45^{\circ}$ angle brackets.

6. Attach Bosch D to Bosch A using the connection explained in step 1. Align the top of $\mathrm{D}$ with the black line on A labeled ' $\mathrm{D}$ '.

7. Attach Bosch E to Bosch A using the connection explained in step 1. Align the top of the Bosch with the black line on A labeled 'E'. The Velcro will be on the bottom side of $\mathrm{E}$.

8. Attach Bosch F to Bosch A. Align the top and bottom of the Bosch with the black lines on A labeled ' $F$ '. The Velcro will be on the bottom side of $F$.

9. Insert a 3/16 screw into the hole on Bosch A labeled ' $S$ '. Match the end hole of the speed light into the screw and orientate to point directly toward the ground. Screw in the speed light until tightly secure.

10. Attach Bosch $G$ to Bosch A. Align the top and bottom of the Bosch with the black lines on A labeled ' $G$ '. The ' $G$ ' label on Bosch $G$ must be oriented toward the back of the tripod.

11. Repeat step 10 for Bosch H.

12. Insert a 3/16 screw into the hole on the end of Bosch E to screw into the hole on the bottom of the camera. Attach the camera to the Velcro on the bottom of E. The camera should point downward in line with E. Screw in the camera until secure.

13. Repeat step 10 with the second camera using the hole on the end of Bosch F.

14. Attach the handle to the top of Bosch A. The handle will attach to A at the uncovered portion of the handle. 
15. Insert a 3/16 screw into the hole in Bosch G. The screw must protrude toward the front of the tripod. Position an LCD monitor with the screen facing upward. Screw in the monitor until secure.

16. Repeat step 13 with a second LCD monitor and Bosch $\mathrm{H}$.

17. Insert the short-cabled battery pack into the right side of Bosch A by placing a $5 / 32$ screw through the hole in the top of the battery pack and slightly attaching a T-nut to the Velcro sid e of the battery pack. Attach with Velcro to A. Make the connection and tighten the screw to secure the T-nut as above.

18. Repeat step 16 using the long-cabled battery pack and the opposite side of Bosch A.

19. Plug the cable from the right side battery pack into the DC plug-in on the left side of the right LCD monitor.

20. Repeat step 17 for the left side battery pack and the left LCD monitor.

\section{Attaching Cables and Well Covers:}

Materials

- Two RCA Video Cables, long

- Two Camera Remotes

- Nine Well Covers

1. Plug one of the video cables into the video connector in the lower camera (the plug-in is next to the memory card slot). Run the cable through the top well of Bosch E and halfway up the front well of Bosch A. Wrap the cable back down toward Bosch E and up to the right LCD monitor. Plug the video cable into the $\mathrm{BNC} / \mathrm{RCA}$ converter on the left side of the monitor.

2. Plug the longer of the two remote cables into the lower camera. Run the cable through the top well of Bosch E and up the front well of Bosch A. Once near the top, Velcro the remote onto the bottom of the handle.

3. Snap the smallest well cover (a thin black strip of plastic with smooth top and two tabs running along the bottom) into the top well of Bosch E to cover the video and remote cables.

4. Plug the other video cable into the upper camera. Run the cable into the top well of Bosch F and coil two times within this well. Plug the other end of the video cable into the BNC/RCA converter on the left LCD monitor.

5. Plug the other remote cable into the upper camera. Run the cable through the right well of Bosch F and attach the back of the remote to the Velcro on the top of the handle.

6. Attach the remainder of the well covers. Well covers are only needed on Bosch F, G, and H. Three well covers will fit on each of three accessible wells on Bosch F. Lay the corresponding well covers over these wells. Each well cover should conceal the entire well. Push each cover into its respective well until it snaps in completely.

7. Repeat step 8 for $\mathrm{G}$ and $\mathrm{H}$. However, only place well covers on the unoccupied top and side wells, leave the bottom wells empty. 
8. Plug the gray cable coming out of the base of the speed light into the plug-in on the front of the camera (next to the gap between the speed light and camera).

9. Once the speed light is plugged in, screw in the silver connector to complete the connection.

\section{Using the Cane Cam:}

1. Turn on the speed light and monitors.

2. Remove the lens caps from the lenses on both cameras.

3. Switch the cameras to Manual mode and press the large button on both remotes to turn on the cameras. The image of the camera will now appear on the LCD monitor. (Nikon Coolpix 995 Settings 3.1.1)

4. The bottom remote controls the lower camera and the upper remote controls the upper camera. (Lower and upper camera resolution: Nikon Coolpix 995 Table1 3.3.1)

5. The cameras are at a different orientation than the monitors. There are small instruction pictures on each monitor to help obtain the correct image. The black arrows represent the direction of tripod movement. The red arrows indicate the direction the image will subsequently move.

6. Loss of battery power in the camera should not be confused with loss of battery power to the LCD monitors. (Nikon Battery 3.1.1) Loss of power to the monitors results in a sudden loss of picture on the screen immediately followed by a blurry, wavy white screen with a mixture of red and blue. Loss of power to the cameras results in a blank screen.

\subsection{Printers}

Printers are helpful in photographic analysis because they can produce a physical copy of an image file. This allows inspectors who are not allowed to remove digital media from the site to print the file and scan it in afterwards to reproduce the file. Also, it creates a backup in case the file is corrupted or lost.

\subsubsection{Generic Ink Jet Printers}

The inkjet printer used for these tests was the HP DeskJet 960C. [10] This printer is approximately the same price as the photo printers tested and produces nearly the same results. (Photo printers 3.2.2) It is the simplest printer to operate and produces quality prints. However, inkjet printers have no exploitable advantages to photo printers in the photo inspection project.

- Inkjet printers in addition to photo printers are an unnecessary means of obtaining hard copies of inspection photographs. 
PNNL-14174

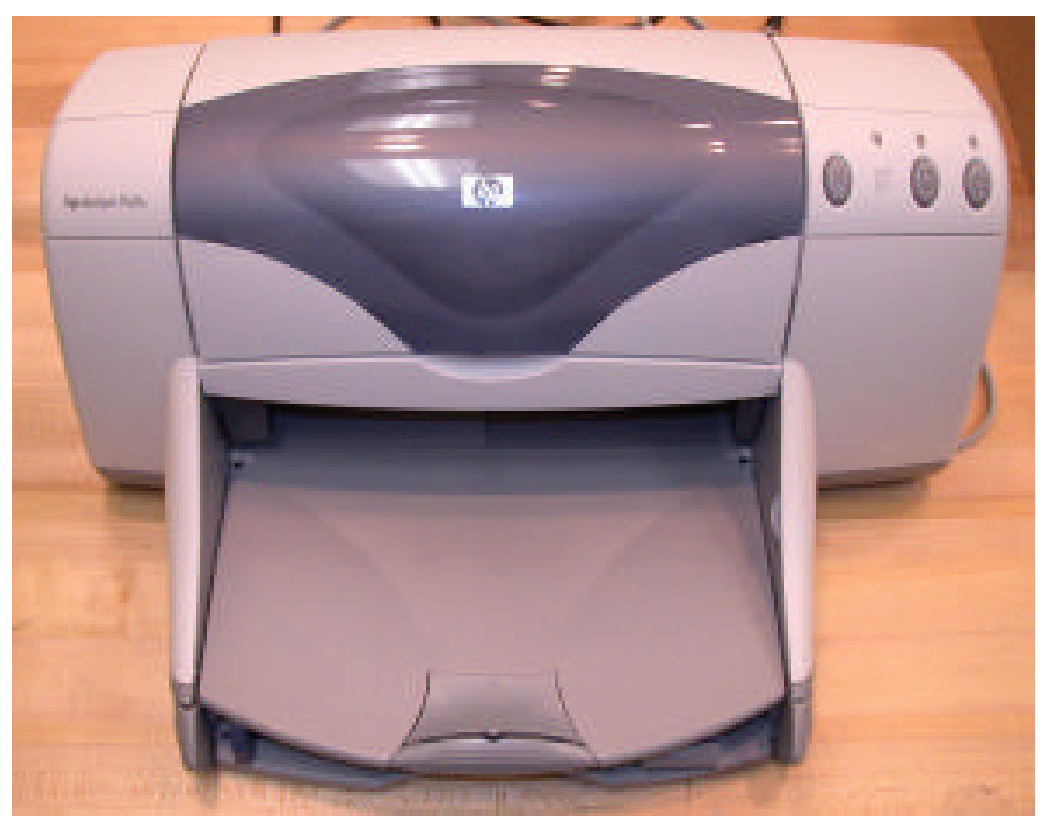

Figure 18: The Hewlett-Packard 960c inkjet printer, an example of a mass-market inkjet printer.

\section{Results}

Maximum dpi: 2400 x 1200 dpi

Usable color dpi: 300 dpi

Resolution Loss- from computer to print: $5-10 \%$

\section{Discussion}

Advantages:

- Ability to print tested scanners' highest dpi (HP Scanners 3.3.1)

Disadvantages:

- Cannot connect directly to digital camera via memory card

- Slow photo printer

\subsubsection{Hewlett-Packard Photosmart Printers}

The photo printers used for these tests were the HP Photosmart 1115 and the HP Photosmart 100. These printers are specifically designed to produce "photograph quality prints". Each has the capability to print directly from a digital camera memory card, with or without an attached computer. There are photo options located on the printer face for simple photo manipulation. 
- Photo printers with memory card print capability are the best option for cost effective, quality photo inspection printing.

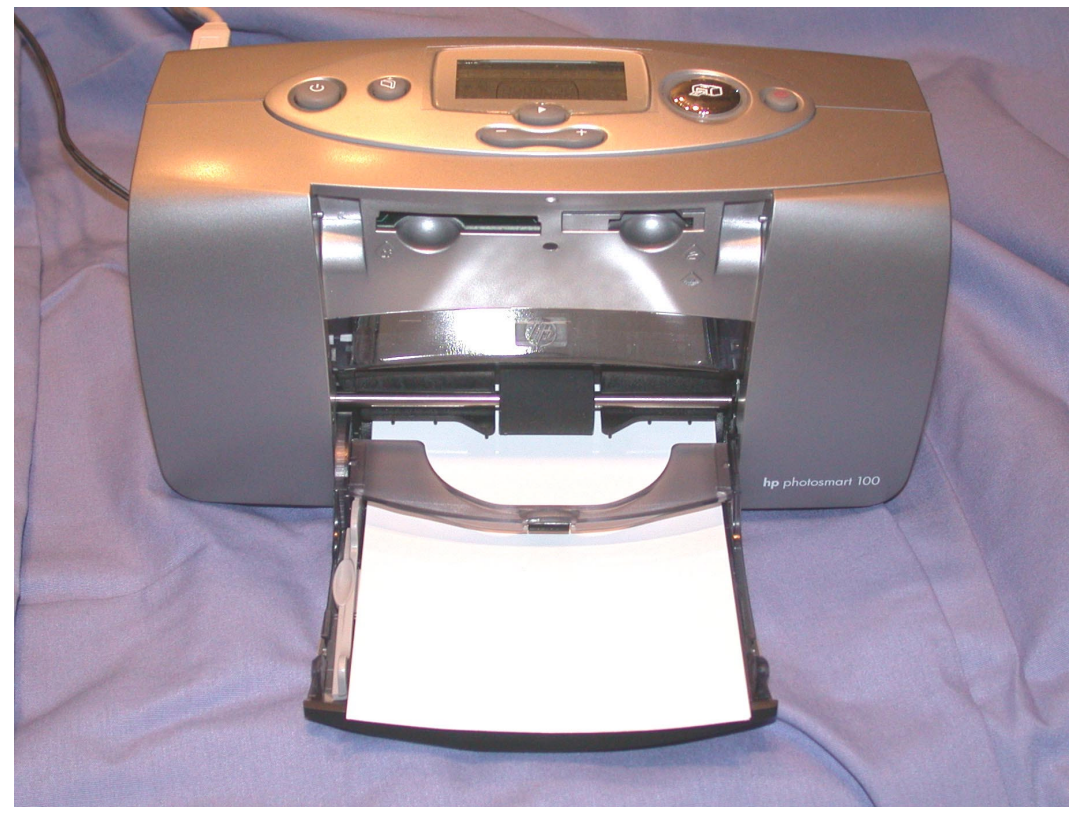

Figure 19: The Hewlett-Packard Photosmart 100 printer, an example of a mass-market printer that prints directly from digital camera storage media.

\section{Results:}

Maximum dpi: 2400 x 1200 dpi

Usable color dpi: $300 \mathrm{dpi}$

Resolution Loss- from computer to print: 5-10\%

\section{Discussion:}

Advantages:

- Ability to print tested scanners' highest dpi (HP Scanners 3.3.1)

- LCD screen with numerous photo options

- Ability to connect directly to digital camera via memory card

- High mobility (HP Photosmart 100)

Disadvantages:

- Slow photo printer 


\subsection{Scanners}

\subsubsection{HP Scanners}

The scanner originally tested for photo-comparison was the HP ScanJet 5300C, a highresolution flat bed color scanner with optical resolution 1200 dpi. An updated version of the ScanJet 5300C, the HP ScanJet 7400C, was tested following use of the ScanJet 5300C. The updated scanner did not actually improve scan resolution as expected. Instead, the ScanJet 7400C merely scanned faster and utilized more advanced HP Precision Scan software. Both scanners are advertised as having a photo quality of 600 dpi (although 200-300 dpi is the actual usable resolution).

- These scanner models provide adequate scanning capability as an accessory for photograph examination.

- There is a significant loss of color quality in a repeated scan and print operation; there should be a color reference in the photograph.

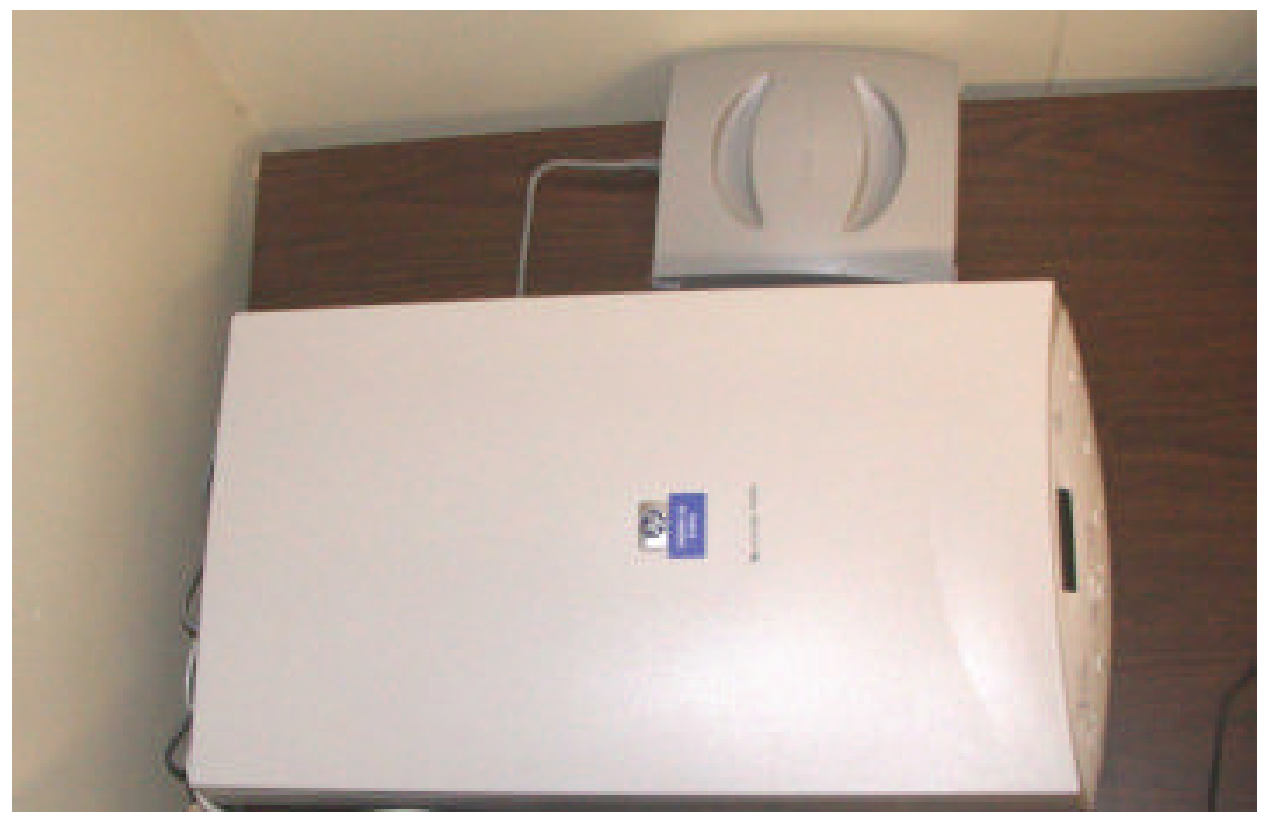

Figure 20: The Hewlett-Packard 7400c scanner (with slide attachment), an example of a massmarket scanner.

\section{Results}

Maximum dpi: 2400 x 1200 dpi (7400C), 1200 dpi (5300C)

Usable dpi: 300 dpi

Resolution Loss- from hard copy to HP Precision Scan: 5-10\%

- from HP Precision Scan to saved file: 5-10\% 
PNNL-14174

\section{Discussion}

\section{$\underline{\text { HP ScanJet 5300C }}$}

Advantages:

- Ability to print directly from Precision Scan

- Manual dpi adjustment

- Simple operation

- Low resolution loss

- 36-bit color

Disadvantages:

- Scans slowly at higher dpi

- Higher dpi does not improve photo quality, 1200 dpi capability is ineffective and useless

- Badly distorts photo color within three rescans

- Scan program may lighten picture when cropping

\section{HP ScanJet 7400C}

Advantages:

- Use of HP Precision Scan 3.02

- Ability to print directly from Precision Scan

- Manual dpi adjustment

- Simple operation

- Low resolution loss

- 48-bit color

- ScanJet XPA (negative scanner) attachment

Disadvantages:

- Higher dpi does not improve photo quality, 2400 x 1200 dpi capability is ineffective and useless

- Badly distorts photo color within two rescans

\subsubsection{Slide Scanners}

There are professional quality slide scanners available to scan large format negatives from 120 and 220 roll film. This service is commonly available from professional photographers; due to the limited production run and high unit cost, these scanners are not mass market consumer items. 


\subsection{Software Tools}

\subsubsection{Photoshop ${ }^{\mathrm{TM}}$ and Color Correction}

Photoshop ${ }^{\mathrm{TM}}$ is a commercial program that allows sophisticated image manipulation. [11] Compared to other programs, it is especially well suited for operations such as precise color matching using a set of reference color tiles such as the GretagMacbeth ColorChecker. [12]

Often the colors in a photograph are altered from their original values through camera and computer processing inaccuracies. If these inaccuracies change between inspections, changes can be found that do not actually exist. Color adjustment is important because it prevents these nonexistent changes from appearing. Adobe Photoshop is the leading picture correction program and includes two ways to manually adjust color levels: color sampling, and manual value adjustment. Color sampling is quick and easy to use, based upon adjusting the RGB (red, green, blue) levels to reproduce known colors such as black and white; manual value adjustment refines color sampling to produce even better color quality in pictures with known colors in the photograph. Both of these methods utilize color tiles to improve photo color and bring each color closer to its true color value. Using the Adobe Photoshop color sampler it is possible to match colors in a picture to obtain precise RGB (red, green, blue) levels, using the procedure recommended for "Digital Publishing". This process works by including ColorChecker tiles at the edge of the photograph, tiles that have known RGB levels as a standard for the picture. Manual adjustment is not perfect however, and a slight disparity may remain between the color values in the picture and the true color values.

The three images below show an unmodified image and the effects of each color adjustment techniques.

\section{Color Adjustment through Color Sampling}

Note: Adobe Photoshop 7.0 was used for these procedures; the commands may be slightly different depending on the version used.

- Open a photograph containing at least a black and white tile in the picture in Adobe Photoshop.

- Select the sampler tool (the eyedropper icon) on the left side of the screen.

- Pull down the drop-down menu Sample Size from below the menu bar.

- Select 3 by 3 Average.

- To open the levels dialog box: Image -> Adjustments -> Levels.

- In the lower right hand corner of the box are three sampler buttons: black, gray, and white.

- Select the white sampler and then click on the white tile in the picture. Follow the same steps for the black sampler and neutral (gray) sampler if a neutral tile is available. Using each sampler will change the color values of the entire picture. 


\section{Color Adjustment through Color Sampling and Value Adjustment}

This method of color adjustment requires a black tile, a white tile, and at least one of the RGB tiles in the picture.

- Select the sampler tool on the left side of the screen.

- To open necessary toolbars: Window -> Options

- Window -> Info

- Pull down the drop-down menu Sample Size from below the menu bar.

- Select 3 by 3 Average.

- To open the levels dialog box: Image -> Adjustments -> Levels.

- In the lower right hand corner of the levels box are three sampler buttons: black, gray, and white.

- Click the white sampler and then click on the white tile in the picture. Repeat for the black sampler and neutral sampler if a neutral tile is available.

- Hold the sampler tool over a RGB tile to find its RGB values.

- Use the slider in the middle of the levels box to obtain values as close to the standard as possible.

- For the first row moving the black slider adjusts the darkness of the blacks in the image, the gray slider adjusts the darkness of colors in between, and the white slider adjusts the darkness of the whites. The first row with three sliders on it controls the levels of each respective color.

- The lower slider works oppositely, the black slider lightens the image as it moves to the right and the white slider darkens the image as it moves to the left.

- Test for correct RGB values by moving the sampler tool over the individual color tiles. 
PNNL-14174

\begin{tabular}{|c|c|c|c|c|}
\hline Number & Name & Red & Green & Blue \\
\hline 1 & Dark skin & 94 & 28 & 13 \\
\hline 2 & Light skin & 241 & 149 & 108 \\
\hline 3 & Blue sky & 97 & 119 & 171 \\
\hline 4 & Foliage & 90 & 100 & 39 \\
\hline 5 & Blue flower & 164 & 131 & 196 \\
\hline 6 & Bluish green & 140 & 253 & 153 \\
\hline 7 & Orange & 255 & 116 & 21 \\
\hline 8 & Purplish blue & 7 & 47 & 122 \\
\hline 9 & Moderate red & 222 & 29 & 42 \\
\hline 10 & Purple & 69 & 0 & 68 \\
\hline 11 & Yellow green & 187 & 255 & 19 \\
\hline 12 & $\begin{array}{l}\text { Orange } \\
\text { yellow }\end{array}$ & 255 & 142 & 0 \\
\hline 13 & Blue & 0 & 0 & 142 \\
\hline 14 & Green & 64 & 173 & 38 \\
\hline 15 & Red & 203 & 0 & 0 \\
\hline 16 & Yellow & 255 & 217 & 0 \\
\hline 17 & Magenta & 207 & 3 & 124 \\
\hline 18 & Cyan & 0 & 148 & 189 \\
\hline 19 & White $\left(.05^{*}\right)$ & 255 & 255 & 255 \\
\hline 20 & $\begin{array}{l}\text { Neutral } 8 \\
\left(.23^{*}\right)\end{array}$ & 249 & 249 & 249 \\
\hline 21 & $\begin{array}{l}\text { Neutral } 6.5 \\
\left(.44^{*}\right)\end{array}$ & 180 & 180 & 180 \\
\hline 22 & $\begin{array}{l}\text { Neutral } 5 \\
\left(.70^{*}\right)\end{array}$ & 117 & 117 & 117 \\
\hline 23 & $\begin{array}{l}\text { Neutral } 3.5 \\
\left(1.05^{*}\right)\end{array}$ & 53 & 53 & 53 \\
\hline \multirow[t]{2}{*}{24} & Black $(1.50 *)$ & 0 & 0 & 0 \\
\hline & $\begin{array}{l}\text { * Optical } \\
\text { Density }\end{array}$ & & & \\
\hline
\end{tabular}

Table 2: True color values for the GretagMacbeth color checker. 
PNNL-14174

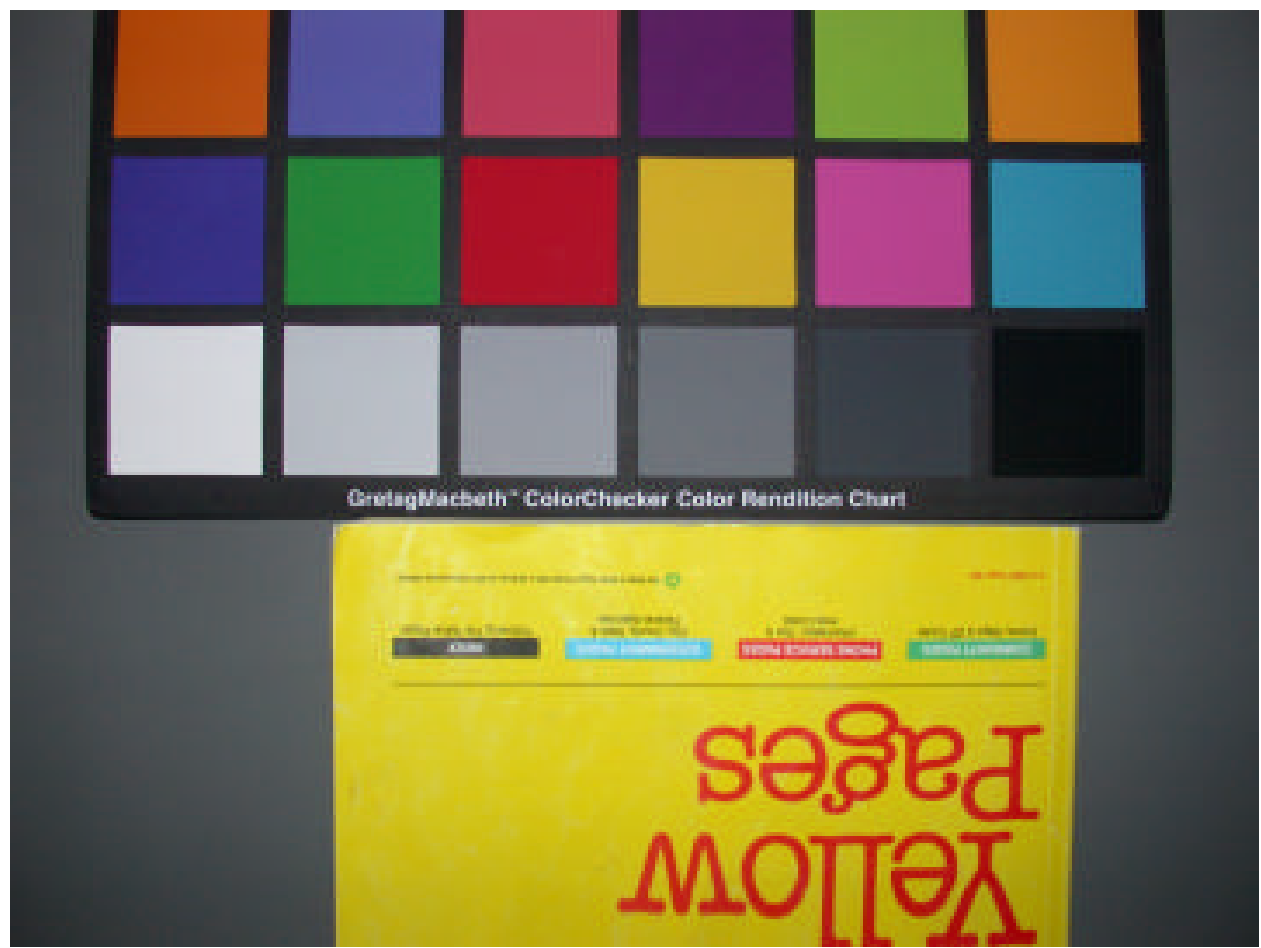

Figure 21: The original image, without modification.

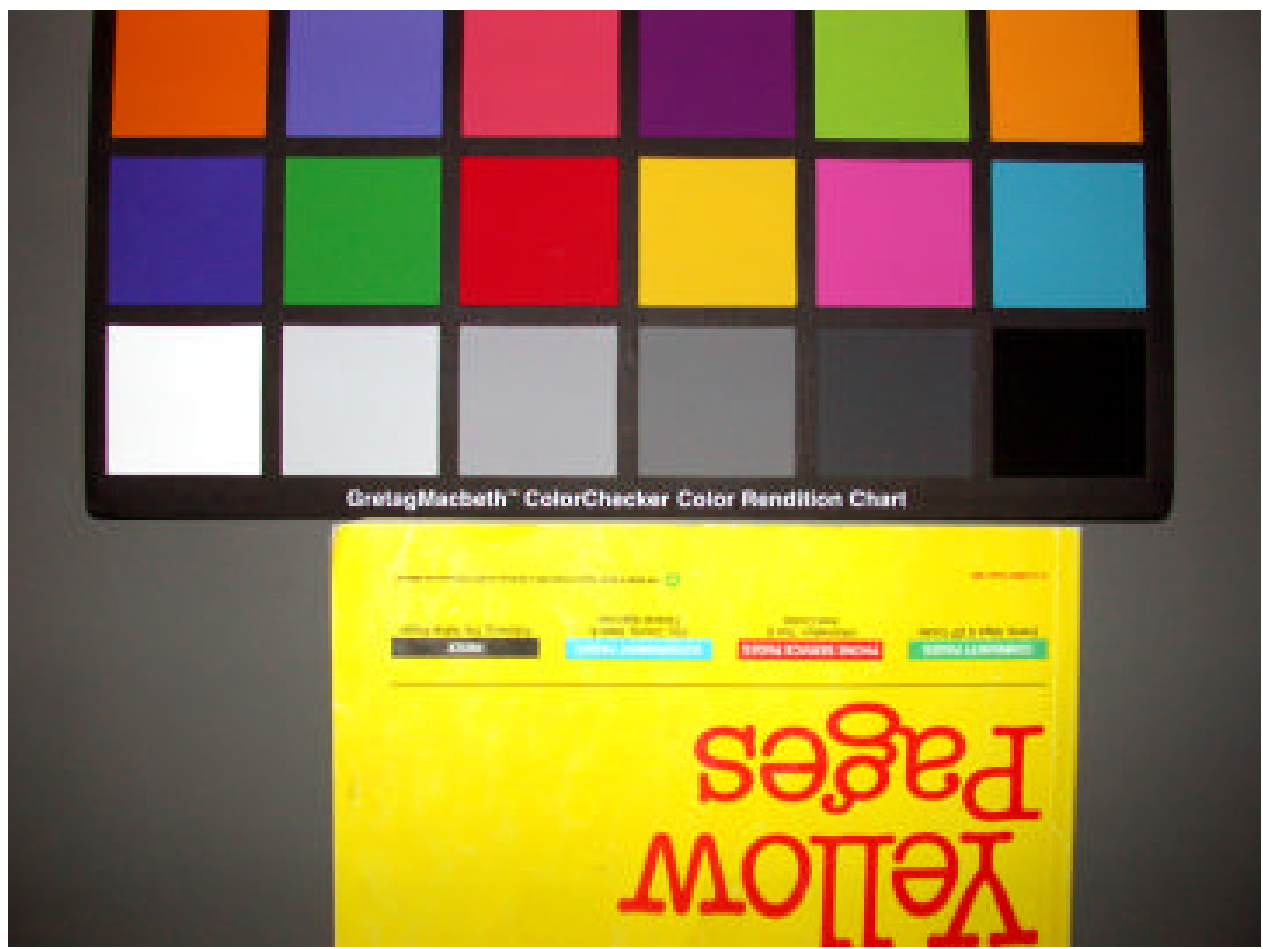

Figure 22: The image adjusted with color sampling of black, white, and neutral (gray). 


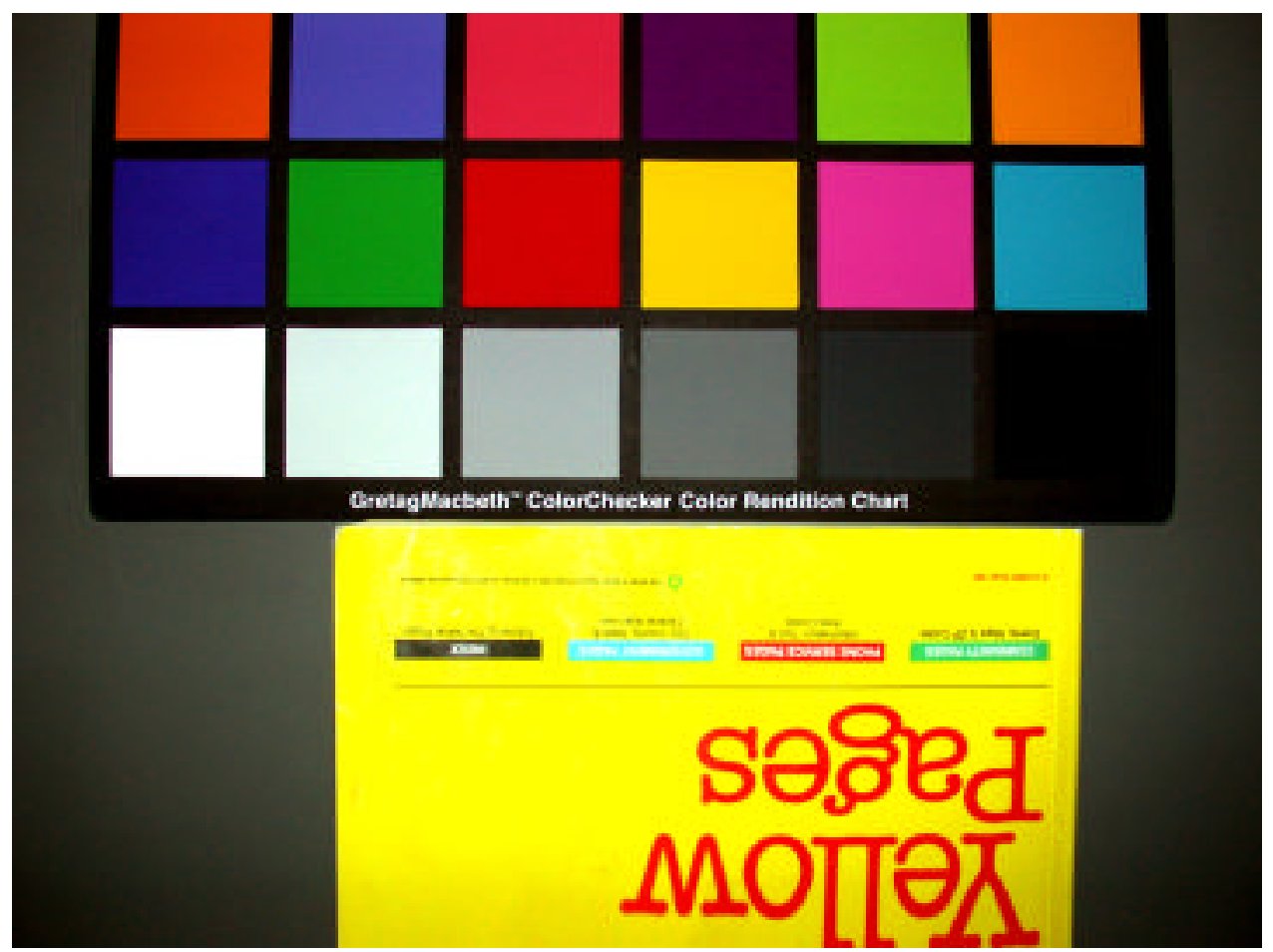

Figure 23: The image adjusted with color sampling and further level adjustment.

\subsubsection{Change Detection System}

The Change Detection System (CDS) is a computer code that takes two images and performs the following actions:

- Fits one image to another, either with or without user supplied fiducials or regions of interest

- Reports the relative overlap between the two as a figure of merit to the fit

- Displays the best fit using a blink comparator feature.

Lassahn, Lancaster, and Jones at the Idaho National Environmental Engineering Laboratory (INEL) developed CDS and allowed its use at PNNL. The PNNL version is version 5.5; development of CDS has continued at the INEL.

The best description of CDS is that it is an adaptive blink comparator. Photographs of everyday objects can be analyzed with blink comparison techniques; however, matching the common features of the two images requires similar image scale and perspective. As long as the two images are sufficiently close, the blink comparison can be carried out with an analysis program that fits one image to the other using standard data fitting techniques. The CDS distorts pairs of digital images to match common features. After fitting, the two images are blink compared. The great advantage of CDS is that it enables blink comparison of photographs from hand-held cameras, as long as the scale and 
perspective of the two photographs are sufficiently similar. One current field application of CDS is the field inspection of cargo containers shipped from the United States to foreign sites, with photographs of the shipped and received container compared before acceptance of the shipment.

\subsubsection{Alternatives to CDS}

Although CDS5 software is an adequate photo-comparison tool, it is important to have alternative methods for finding differences between circuit boards or other tamper deterrent features. One reason to investigate alternatives is that the CDS software is not a commercial product capable of procurement in foreign countries. In addition, change comparison is computationally intensive and a faster program would be advantageous. Companies suggested with potential programs for these purposes included: Braintech, Media Cybernetics, and Image Source. Several programs already in use at the laboratory such as Adobe Photoshop, IDL, Microsoft Power Point, and Mathematica were also considered. The objective was to find software with the potential to overlay two images, then stretch, shrink, and orientate the two images for optimal comparison.

Pixel-to-Pixel Comparison: There were two programs that could perform pixel-to-pixel comparison: ENVI by Research Systems, and Mathematica by Wolfram. [13,14] Pixelto-pixel comparisons can be effective if the two pictures being compared are in the same position and orientation. However, detection ability quickly fades as the two pictures become increasingly different in these two aspects.

- ENVI does allow for determination of the amount of pixels a pixel on the original picture is away from the same pixel on the comparison picture. However, this matching process creates a futile guessing game

- Mathematica software allows pixel-to-pixel comparison by selecting pixels to align the images, which will not overlap. However, this program is overly difficult to operate, and is not worthwhile for use.

Neither program is a viable alternative to CDS.

Color Curve Comparison: The Adobe Photoshop software had two methods for comparing photographs. One option is examination of two photos side by side or on top of one another using zoom or pan for alternate views, the second option is color curve adjustment. (Photoshop Color Level Adjustment 3.4.2) Modifying the color curve of the images enhances changes by altering the way that each color is represented on the screen. Creating a color curve with one of the pictures being compared and loading it on to the other picture manifests changes to many distinctly colored features (burns, solder bumps, solder dust, etc.). The procedure is

- In Adobe Photoshop, open the curves dialog box on the first picture: Image -> Adjustments -> Curves

- Modify the curve in the grid until photo shows a mix of colors sufficient for comparison (usually two curve changes). 
- Save the curve and close dialog box

- Open the comparison picture.

- Reopen the curves dialog box

- Load the saved curve on new picture

Adobe Photoshop is a good choice for simple, easy to spot changes. Although once changes become more intricate, Photoshop's usefulness diminishes.

Simple Comparison: One of the simplest methods for photo comparison was the use of any slideshow program such as Microsoft Power Point. By creating a slideshow with two slides set to run in an infinite loop the pictures will continuously flash back and forth. The major problem with this type of software is that the pictures must be almost perfectly aligned for successful change detection.

- Slideshow programs are the easiest and quickest programs to use if the pictures are scaled and in the same position

- This would require photography from a fixed geometry.

\section{Summary and Conclusions}

This study has demonstrated that recent advances in mass-market photographic equipment and computer analysis packages enable a collection of items to be archived and monitored for changes through tamper indicating devices and photographic analysis. The representative samples in this study illustrate the utility of even these relative simple techniques that are easily implemented. Future development activity should concentrate upon a study of other tags and seals, such as the E-cup or Cobra seal, and representative examples of enclosures and doors.

This project was supported by the Defense Threat Reduction Agency, an agency of the US Department of Defense. PNNL is operated by Battelle for the United States Deparament of Energy under Contract DE-ACO6-76RL0183O. 
PNNL-14174

\section{References}

[1.] Dennis di Cicco, "Hunting Asteroids", CCD Astronomy, Volume 1, Number 2, 8-13, Spring 1996. An abridged version is available from the Sky and Telescope webpage: http://skyandtelescope.com/printable/objects/asteroids/article_378_1.asp

[2] Gordon D. Lassahn, James L. Jones, and Gregory D. Lancaster, Idaho National Environmental Engineering Laboratory. A general description of the CDS can be found in New Scientist, Volume 171, Number 2309, p. 22, 2001

[3] Representative papers include "An Annotated Taxonomy of Tag and Seal Vulnerabilities", Roger G. Johnston and Anthony R.E. Garcia, in The Journal of Nuclear Materials Management, Volume 28, p 23-30, Spring 2000; "Efficacy of TamperIndicating Devices", Roger Johnston, Anthony R.E. Garcia, and Adam N. Pacheco, LAUR-02-0492; "Domestic vs. International Safeguards", Roger G. Johnston and Morten Bremer Maerli, LAUR-01-5365; "Tamper-Indicating Seals for Nuclear Disarmament and Hazardous Waste Management", Roger G. Johnston, in Science and Global Security, Volume 9, pp. 93-112, 2001; and "Were ancient seals secure?", Roger Johnston, Debbie D. Martinez, and Anthony R.E. Garcia, in Antiquity, Volume 75, pp. 299-305, 2001

[4] B.W. Wright and H.A. Undem, "Tamper Tape Seals", presented at the $35^{\text {th }}$ Annual Meeting of the Institute for Nuclear Materials Management, July 17-20, 1994, Naples, FL. Available by request from Pacific Northwest National Laboratory (report PNL-SA23817) or from the Department of Energy's "Information Bridge" electronic archive, www.osti.gov/bridge

[5] 3M Corporation, Safety and Security Systems Division, 1872 Neal Avenue South, Afton, MN 55001, www.3m.com

[6] Nikon USA, 1300 Walt Whitman Road, Melville, NY 11747, http://www.nikonusa.com

[7] Mamiya America Corporation, 8 Westchester Plaza, Elmsford, NY 10523, http://www.mamiya.com

[8] Polaroid Corporation, 1265 Main Street - Bldg. W3, Waltham, MA 02451, www.polaroid.com

[9] Thales Optem, 78 Schuyler Baldwin Drive, Fairport, New York, 14450-9196 www.thales-optem.com

[10] Hewlett-Packard, 3000 Hanover Street, Palo Alto, CA 94304-1185, www.hp.com

[11] Adobe Systems Incorporated, 345 Park Avenue, San Jose, California 95110-2704, www.adobe.com 
[12] Edmund Industrial Optics, 101 East Gloucester Pike, Barrington, NJ 08007-1380, www.edmundoptics.com

[13] Research Systems, Inc., 4990 Pearl East Circle, Boulder 80301, www.rsinc.com

[14] Wolfram Research, Inc., 100 Trade Center Drive, Champaign, IL 61820, www.wolfram.com 